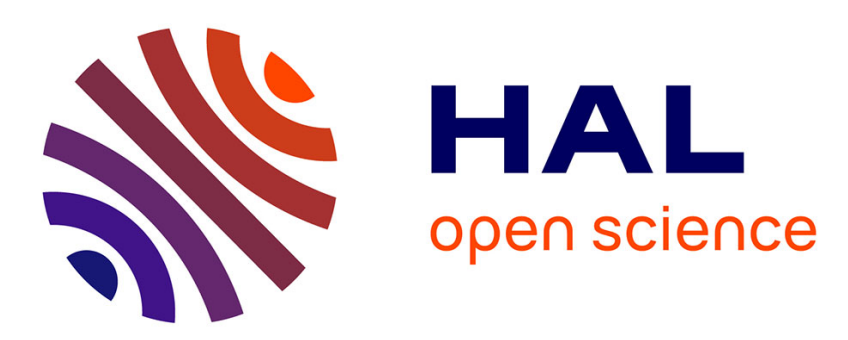

\title{
On the Efficiency of Local Electricity Markets Under Decentralized and Centralized Designs: A Multi-leader Stackelberg Game Analysis
}

Hélène Le Cadre

\section{To cite this version:}

Hélène Le Cadre. On the Efficiency of Local Electricity Markets Under Decentralized and Centralized Designs: A Multi-leader Stackelberg Game Analysis . 2018. hal-01619885v2

\section{HAL Id: hal-01619885 \\ https://hal.science/hal-01619885v2}

Preprint submitted on 4 Jan 2018

HAL is a multi-disciplinary open access archive for the deposit and dissemination of scientific research documents, whether they are published or not. The documents may come from teaching and research institutions in France or abroad, or from public or private research centers.
L'archive ouverte pluridisciplinaire HAL, est destinée au dépôt et à la diffusion de documents scientifiques de niveau recherche, publiés ou non, émanant des établissements d'enseignement et de recherche français ou étrangers, des laboratoires publics ou privés. 


\title{
On the Efficiency of Local Electricity Markets Under Decentralized and Centralized Designs: A Multi-leader Stackelberg Game Analysis*
}

\author{
Hélène Le Cadre ${ }^{\dagger}$
}

\begin{abstract}
In this paper, we analytically compare centralized and decentralized market designs involving a national and local market operators, strategic generators having market power and bidding sequentially in local markets, to determine which design is more efficient for the procurement of energy. In the centralized design, used as benchmark, the national market operator optimizes the exchanges between local markets and the generators' block bids. In the decentralized design, generators act as Stackelberg leaders, anticipating the local market prices and the flows on the transmission lines. Clearing of the local markets can be either simultaneous or sequential. The resulting two-stage game with competitive leaders that are not price takers is formulated as a bilevel mathematical programming problem which is reformulated as a Nash-Cournot game, and conditions for existence and uniqueness of market equilibrium are studied. Imperfect information is also considered, resulting from the lack of incentives from the generators to share their RES-based generations. Through a case study, we determine that the decentralized design is as efficient as the centralized one with high share of renewables, using as performance measure the Price of Anarchy, and that imperfect information has a limited impact on the efficiency of the decentralized market design. Furthermore, we check numerically that there exists an upper-limit on the block bid length maximizing the social welfare under both centralized and decentralized designs.
\end{abstract}

Keywords: Bilevel Mathematical Programming; Complementarity Theory; Electricity Market; Bidding; Price of Anarchy

\section{Introduction}

The development of Distributed Energy Resources (DERs) may offer new sources of flexibility, which may allow system operators to cost-effectively mitigate the im-

\footnotetext{
${ }^{*}$ The author would like to thank the two anonymous referees for their feedback, which have greatly contributed to the improvement of the article content.

${ }^{\dagger}$ VITO/EnergyVille research center, Thor scientific Park, Genk, Belgium. Email: helene. lecadre@energyville.be
} 
pact of variable and unpredictable generation from Renewable Energy Sources (RES). However, contracting DER-based generation in a centralized market structure may complicate market clearing procedures due to the amount and complexity of the bids and the associated coordination requirements between market parties [18]. The goal of this article is to compare the efficiency of two market designs for energy procurement with rebalancing: a centralized market, coordinated by a single national Market Operator (MO), and a decentralized market, governed by a number of strategic generators located in local markets, that interact with the national MO.

In a centralized energy market, a coordinating MO contracts at the national level DER directly from DER owners connected to the distribution grid, possibly by the intermediate of an aggregator. Such a centralized market can be formulated as a standard optimization problem under transmission network constraints. On the contrary, in a local market structure, a MO at the local (e.g., distribution) level matches DERbased generation and local demand. In these local markets, generators have market power, i.e., they adjust their quantities, recognizing that they can affect market clearing prices $[4,36]$. In this two-settlement model, generators anticipate the national market clearing and behave as local leaders. Interactions between local markets may be managed by a national MO. As a consequence, resources connected to the distribution grid can only be offered to a global (national) market via local markets, taking into account the transmission network constraints. Local generators will be considered as multiple leaders who anticipate the reaction of the national MO who behaves as a follower. Such hierarchical market structures, in which agents have conflicting interest while sharing network constraints belong to the stream of literature called networked Stackelberg competition [2, 3, 35]. Failures observed in electricity market competition literature that increase the need for introducing a centralized authority (either a national Market Operator or a regulator) to coordinate the involved agents, do not seem to be specific to the energy sector. Similar observations were made for example in communication networks, and more specifically, in the Internet, where service providers and content providers interact through a jointly shared network infrastructure. In the Internet economy, to avoid failures resulting from service providers' discriminatory behavior, the Network Neutrality principle was introduced to guarantee the equal treatment of all the data streams by service providers [10].

In this article, we consider a stylized energy market with rebalancing (i.e., exchanges between the local markets) where the bids take the form of either simple quantity offers or block quantity offers that are subject to inter-temporal constraints [28]. We assume that these inter-temporal constraints are set up so as to define a convex feasible region for the bids, to keep the problem analytically tractable. In actual markets, start up costs, block bids with minimum income requirements, load gradient, exclusive orders, etc. [28, 39], render the generators' optimization problems highly non-convex and are thus not amenable to an analytical solution.

We also take into account the transmission network through a simplified linear DC power flow model, which represents an approximation of Kirchhoff's laws. This assumption is classical and well-accepted in the electrical engineering literature [5, 23, $29,33]$. In practice, flows on lines can be calculated using power transfer distribution factor which specifies the proportion of flow on line resulting from an injection of one unit electricity at the corresponding source node and a corresponding one unit withdrawal at some fixed reference node [36]. Distribution level constraints are ignored in the provision of DER-based generation.

Stackelberg games are hierarchical games involving a leader and a follower. The leader takes the followers' optimal reaction into account when optimizing his strategy. 
Such games are traditionally formulated as bilevel optimization problems $[6,7,8,17]$. Dedicated reformulations are required to solve these multi-leader follower games [9]. In a first stream of the literature, such problems are reformulated using the Karush-KuhnTucker conditions for the lower level problems resulting in a Mathematical Program with Complementarity Constraints (MPCC) [7, 9, 12]. However, as pointed out by Dempe and Dutta in [9], such reformulation may not coincide with solutions of the original Stackelberg game. In the non-convex cases, the Karush-Kuhn-Tucker conditions are not sufficient and MPCC reformulation will result in a much larger feasible set. If the lower-level is a parametric convex optimization problem, well developedapproaches are used to compute local optima that in general do not coincide with equilibrium of the Stackelberg game, unless the Slater's constraint qualification is checked at the lower level [9]. Another stream of the literature on bilevel programming, reformulates the lower-level problem as a variational inequality [6]. Variational inequalities are mathematical programs that allow the modelling of many equilibrium phenomena encountered for example, in complementarity games in energy [12]. Mathematical Programs with Equilibrium Constraints (MPECs) may be viewed as bilevel programs where the lower-level problem consists in a variational inequality. Both streams have generated an abundant literature in the community of mathematical bilevel optimization [6, 7, 8], and game theory for energy problems [12].

Equilibrium Problems with Equilibirum Constraints (EPECs) are generalizations of MPECs in which multiple Stackelberg leaders participate in a simultaneous game, the follower taking the strategies of the leaders as given and leaders making decisions subject to the equilibrium conditions arising from the follower lower-level optimization problem [17]. EPEC solving is still considered as a challenging problem in the optimization and game theory communities [17]. However, several applications of multi-leader follower games can be found in the energy literature [12, 23]. In [19], we provided a game theoretic based representation of suppliers that interact in geographic demand markets, organized as two-tiered systems. Assuming rational expectation of the agents with respect to the outcome of the real-time market, existence and uniqueness of subgame perfect Nash equilibrium are investigated. Strategic behaviors of the agents have also been considered in $[13,30]$. In these papers, the authors considered respectively a strategic power producer and an aggregator that bid into the day-ahead electricity market with the objective to maximize their profit and minimize charging costs respectively, while anticipating the market clearing, behavior and data of rival producers and consumers. In [23], Neuhoff et al. provide a very complete review of numerical models of transmission-constrained electricity markets. In Cambridge II and Madrid models, an integrated transmission and energy market is introduced $[1,4,11]$ where leaders are not price takers: each Stackelberg leader (strategic generator) anticipates what happens to locational marginal prices, as well as flows on the transmission lines. This is strongly related to the formulation that we present in this paper, except that we do not consider fringe generation and that we introduce sequential bidding which complexifies the computation of market equilibrium. Furthermore, we propose an analytical approach to derive conditions for existence and uniqueness of market equilibria, that can be seen as complementarity to the numerical models introduced in [23].

In this paper, we propose an analysis of a multi-leader Stackelberg game under generator market power and convex cost functions. We bring contributions to the existing literature according to three aspects that we detail below:

(i) a first contribution relies on the formal proofs of the efficiency of decentralized 
energy markets compared to the classical centralized energy market which maximizes social welfare, and on the effect of sequential bidding, when generators have market power and are not price takers.

(ii) a second contribution is related to the inclusion of imperfect information in the two-settlement market model.

(iii) a third contribution is an analytical comparison of sequential versus simultaneous local market clearing.

The article is organized as follows. We start by introducing the model in Section 2, i.e., the network model in Subsection 2.1, the agents and the utility functions in Subsections 2.2 and 2.3 respectively, and the solution concepts that will be used throughout the article in Subsection 2.4. Then we provide a mathematical model for centralized and decentralized market designs in Subsections 3.1 and 3.2. We characterize analytically the existence and uniqueness of solutions in each of these market designs in Subsection 3.3, and quantify the efficiency of decentralized markets (cleared either simultaneously or sequentially) with high shares of renewables, with respect to the centralized market design using the Price of Anarchy as performance measure (Subsection 4.1). Finally, a case study is provided in Section 4 to quantify the impact of the share of renewables on the Price of Anarchy and on the existence of subgame perfect Nash equilibrium for the decentralized market design, taking into account intertemporal constraints. A last numerical illustration opens the discussion towards the necessity to find a trade-off between increasing bid complexity and guaranteeing the efficiency of the market operation.

\section{Notation}

To uniformize the notations, vectors and matrices will be denoted by bold characters.

Agents
\begin{tabular}{|c|c|}
$G_{k}$ & conventional generator in local market $k$ \\
$M O_{k}$ & local Market $k$ Operator \\
$M O$ & national Market Operator \\
\hline
\end{tabular}

Mathematical operators
\begin{tabular}{|c|c|}
$\boldsymbol{x}^{T}$ & transpose of vector $\boldsymbol{x}$ \\
$\mathbf{1}$ & unitary vector \\
$\nabla \mathcal{J}$ & gradient of multi-variable function $\mathcal{J}$ \\
\hline
\end{tabular}

\begin{tabular}{|c|c|} 
Parameters & generic time period \\
$t$ & time horizon \\
$t_{H}$ & number of local markets \\
$n$ & number of edges \\
$l$ & line transmission capacities \\
$\boldsymbol{f}$ & shift-factor matrix \\
$\boldsymbol{H}$ & price parameters in local market $k$ \\
$a_{k}, b_{k}$ & information distortion factor in local market $k$ \\
$\xi_{k}$ &
\end{tabular}




\begin{tabular}{|c|c|} 
Variables & local market $k$ simple bid at time period $t$ \\
$q_{k}(t)$ & simple bids of all the other local markets except $k$ at $t$ \\
$\boldsymbol{q}_{-\boldsymbol{k}}(\boldsymbol{t})$ & block bid in local market $k$ \\
$\boldsymbol{q}_{\boldsymbol{k}}$ & vector of simple bids at time period $t$ \\
$\boldsymbol{q}(\boldsymbol{t})$ & net power injection into local market $k$ node at time period $t$ \\
$-r_{k}(t)$ & vector of exchanges between local markets at time period $t$ \\
$\boldsymbol{r}(\boldsymbol{t})$ & RES-based generation in local market $k$ at time period $t$ \\
$w_{k}(t)$ & estimated RES-based generation in local market $k$ at $t$ \\
$\hat{w}_{k}(t)$ & vector of the estimated local RES-based generations at $t$ \\
$\hat{\boldsymbol{w}}(\boldsymbol{t})$ & demand in local market $k$ at time period $t$ \\
$d_{k}(t)$ & locational marginal price in local market $k$ \\
$p_{k}()$. & Lagrange multiplier \\
$\boldsymbol{\lambda}$ & aggregated demand parameters \\
$A, B$ & optimal exchanges under centralized design \\
$r^{c}(\hat{\boldsymbol{w}})$ & $G_{k}$ 's optimal production for the centralized design \\
$q_{k}^{c}(\hat{\boldsymbol{w}})$ & $G_{k}$ 's optimal bid for the decentralized design \\
$q_{k}^{\star}(\boldsymbol{w})$ & total conventional and renewable generation \\
$Q$ &
\end{tabular}

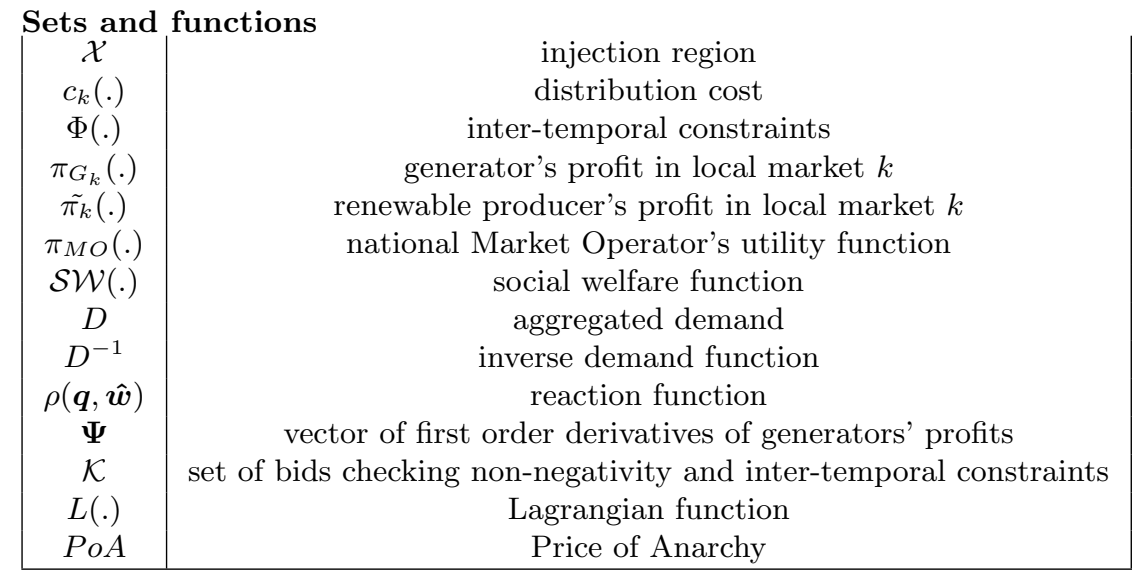

\section{The model}

In this section, we describe the network model in Subsection 2.1, the agents and the utility functions in Subsections 2.2 and 2.3 respectively, and the solution concepts that will be used through the article in Subsection 2.4 .

\section{$2.1 \quad$ Network model}

We consider a power network with $n$ nodes (also called buses in electrical engineering) labeled $\{1, \ldots, n\}$ and $l$ edges (lines). Each node will be associated with a local market. The line flows are related to the nodal power injections through Kirchhoff's laws.

The national Market Operator (MO) controls the exchanges between local market nodes $\boldsymbol{r}(\boldsymbol{t})$ and the corresponding locational marginal prices are either fixed by the national MO in case where a centralized market is implemented, or by local MOs in case where a decentralized market holds. The exchanges between local market nodes 
$\boldsymbol{r}(\boldsymbol{t})$ must satisfy the network feasibility constraints, that is, the resulting power flows should not exceed the transmission line capacity $f$ in both directions. We model the transmission network through a simplified linear DC power flow model, which represents an approximation of Kirchhoff's laws. This assumption is classical and well-accepted in the electrical engineering literature [5, 23, 29, 33]. In practice, flows on transmission lines can be calculated using power transfer distribution factor which specifies the proportion of flow on one line resulting from an injection of unit electricity at the corresponding source node and a corresponding one unit withdrawal at some fixed reference node [36]. In our network model, distribution level constraints are ignored in the provision of DER-based generation. On top of that, because we do not consider storage in this paper, the demand and generation must be balanced at every time period, implying that all exchanges between local market nodes must sum up to zero.

Formally, all these constraints are captured through the injection region $\mathcal{X}$ that we define below:

$$
\mathcal{X}:=\left\{\boldsymbol{x} \in \mathbb{R}^{n} \mid-\boldsymbol{f} \leq \boldsymbol{H} \boldsymbol{x} \leq \boldsymbol{f}, \mathbf{1}^{T} \boldsymbol{x}=0\right\},
$$

where the matrix $\boldsymbol{H}$ is known as the shift-factor matrix that depends on the admittances of the transmission lines of the power network [37]. The transmission capacities of the lines are given by $\boldsymbol{f} \in \mathbb{R}_{+}^{l}[36]$.

\subsection{Agents}

We consider a local networked market that is cleared at each time period $t \in\left\{0, \ldots, t_{H}-\right.$ $1\}$ over a finite time horizon $t_{H} \in \mathbb{N}^{*}$, the local MO taking into account local network constraints, and on which conventional generators, consumers and renewable producers interact. The local MO reports the cleared quantities to the national MO who is responsible for the operation of the global (national) market.

We consider quantity offers that are bidded on the energy market and take the form of (linked) block bids, i.e., length $t_{H}$ vectors subject to inter-temporal constraints that are accepted in full. In the EU Power Exchange context, generators have the possibility to submit a certain interval of consecutive hours where they are willing to produce [27]. In our paper, these inter-temporal constraints are set up so as to define a convex feasible region for the bids, the goal being to keep the problem analytically tractable to obtain closed form expressions for market equilibrium. In Section 4 we will quantify the impact of parametrizations of the feasible set on the existence of market equilibrium. These aspects have already been pointed out in [3, 12], as well as the fact that mixed strategy equilibria exist where pure strategy ones might not. In actual markets, start up costs, block bids with minimum income requirements, load gradient, exclusive orders, etc. [28, 39], render the generators' optimization problems highly non-convex and are thus generally not amenable to an analytical solution. An alternative is to use simple bids, which does not require to introduce inter-temporal constraints. But, taking an operational perspective, this means that, for instance, the offers of one thermal generating unit could be accepted in the 3-rd, 5-th, and 7th periods, leading to a unit schedule which could be highly uneconomical or simply infeasible from the technical perspective. The simple idea behind the introduction of inter-temporal constraints is to introduce as few complex constraints as possible in the bid, so as not to complicate the matching process in excess while at the same time removing the huge risk at which agents are exposed with simple bids. So inter-temporal constraints in the bids may capture a mixed effect of actual operational constraints 
(start up costs, ramping constraints) and cost components.

We introduce the definition of a bijective application, which will be used to define admissible inter-temporal constraints on the block bids:

Definition 1. The application $\Phi: \mathbb{R}_{+}^{t_{H}} \rightarrow \mathbb{R}^{m}$ is bijective if, and only if, $\forall \boldsymbol{y} \in \mathbb{R}^{m}$, there exists a unique $\boldsymbol{q} \in \mathbb{R}_{+}^{t_{H}}$ such that $\Phi(\boldsymbol{q})=\boldsymbol{y}$.

We now describe in more details the various categories of agents that interact in the market place:

- Local Market Operator (MO): in each local market node $k \in\{1, \ldots, n\}$ and at each time period $t \in\left\{0, \ldots, t_{H}-1\right\}$, there is a local MO that clears the local market which is composed of one aggregated conventional (nuclear, gas, coal, etc.) generator $G_{k}$, one aggregated renewable producer and consumers.

- Conventional generators: $G_{k}$ submits a quantity offer $\boldsymbol{q}_{\boldsymbol{k}} \in \mathbb{R}_{+}^{t_{H}}$ and incurs a production cost $\sum_{t=0}^{t_{H}-1} c_{k}\left(q_{k}(t)\right)$ for $\boldsymbol{q}_{\boldsymbol{k}}$, where the cost function $c_{k}: \mathbb{R}_{+} \rightarrow$ $\mathbb{R}_{+}$is assumed to be continuously differentiable, strictly increasing, and convex with $c_{k}(0)=0$. The block bids of the generators are subject to inter-temporal constraints $\Phi\left(\boldsymbol{q}_{\boldsymbol{k}}\right) \leq \mathbf{0}$ where $\Phi: \mathbb{R}_{+}^{t_{H}} \rightarrow \mathbb{R}^{m}$ is continuous, bijective following Definition 1 and increasing in $\boldsymbol{q}_{\boldsymbol{k}}$ and $m \in \mathbb{N}^{*}$ is the number of inter-temporal constraints that are considered.

- Renewable producers: local MOs may also have RES-based generation in their portfolio, and take it into account when clearing the local markets. We assume a zero marginal cost for the RES-based generation and let $\hat{w}_{k}(t)$, be the estimated RES-based generation in market $k \in\{1, \ldots, n\}$ node, at time period $t \in\left\{0, \ldots, t_{H}-1\right\}$.

- Consumers: we assume that the aggregated consumption in each local market node $k$ is represented with an inverse demand function $p_{k}: \mathbb{R}_{+} \rightarrow \mathbb{R}_{+}$that specifies how much the aggregate consumer is willing to pay to consume one more unit of electrical energy. $d_{k}(t)$ is the aggregated demand in market node $k \in\{1, \ldots, n\}$, at time period $t$. The consumer inverse demand function $p_{k}($.$) is$ assumed to be twice continuously differentiable, strictly decreasing, and concave, i.e., $p_{k}^{\prime}()<$.0 and $p_{k}^{\prime \prime}() \leq$.0 . We assume a linear relation between price and aggregated demand leading to:

$$
p_{k}\left(d_{k}(t)\right)=a_{k}-b_{k} d_{k}(t)
$$

for some $a_{k}>0$ and $b_{k} \geq 0$.

Regarding the use of local renewable production, two cases are possible:

(i) $d_{k}(t)<\hat{w}_{k}(t)+q_{k}(t)$ there is a surplus of power which is bought by the local MO on the local market and submitted to the national MO on the global market,

(ii) $d_{k}(t) \geq \hat{w}_{k}(t)+q_{k}(t)$ there is a lack of power that needs to be bought by the local MO.

Conventional and renewable generators will be aggregated in a single portfolio, in each local market. 
- National Market Operator (MO): the global (national) MO determines the exchanges between different market nodes $\mathbf{r}(\mathbf{t}) \in \mathcal{X}^{n}$. Note that for all $k \in$ $\{1, \ldots, n\},-r_{k}(t)$ denotes the net injection into market node $k$, at time period $t$. These exchanges are determined according to a market mechanism that maximizes the social welfare while guaranteeing supply and demand balance in each market node. Exchanges, $\mathbf{r}(\mathbf{t})$, are constrained by the available transmission capacity between the market nodes.

\subsection{Timing of the game and utility functions of the agents}

Demand and supply balance at each node $k \in\{1, \ldots, n\}$ and each time period $t \in$ $\left\{0, \ldots, t_{H}-1\right\}$ implies that:

$$
d_{k}(t)=q_{k}(t)+\hat{w}_{k}(t)+r_{k}(t)
$$

which implies in turn that $p_{k}\left(d_{k}(t)\right)=p_{k}\left(q_{k}(t)+\hat{w}_{k}(t)+r_{k}(t)\right)$. We model a nodal pricing mechanism which properly rewards DERs. Exchanges between local markets are only coming from consumers. The national MO plays on it by changing the price so that consumers change how much they buy/consume. This is similar as the approach introduced in [36] where the demand side is price taking with elastic demand functions subject to quantity shifts in each local market ${ }^{1}$.

Depending on the market design, the strategic interactions between the agents is either modeled as a standard constrained optimization problem (centralized market) or as a Stackelberg game (decentralized market). The Stackelberg game takes place as follows:

1) each conventional generator $G_{k}, k \in\{1, \ldots, n\}$ chooses independently and simultaneously his block bid $\boldsymbol{q}_{\boldsymbol{k}}$ to maximize his profit, anticipating the sequence of local market clearing prices and exchanges between local market nodes.

2) at each time period $t \in\left\{0, \ldots, t_{H}-1\right\}$, each local MO clears the local market and announces the price $p_{k}\left(q_{k}(t)+\hat{w}_{k}(t)+r_{k}(t)\right)$ to the conventional generator $G_{k}$ and the consumers. Subsequently, the central MO chooses the exchanges between markets, $\boldsymbol{r}(\boldsymbol{t})$, that maximize the global social welfare.

With decentralized market design, the prices at different locations can differ. This setting follows the locational marginal pricing approach [26], used commonly in the $\mathrm{US} \mathrm{system}^{2}$, and strongly related to Yao et al. formulation in [36].

With the centralized market design, we assume that there is no anticipation at the generators' side on the exchanges between local markets and that the national MO optimizes simultaneously the production quantities $\left(\boldsymbol{q}_{\boldsymbol{k}}\right)_{k=1, \ldots, n}$ and the exchanges between local markets $(\boldsymbol{r}(t))_{t=0}^{t_{H}-1}$ in order to maximize the social welfare.

We now detail the utility functions of the agents involved in the Stackelberg game. At time period $t \in\left\{0, \ldots, t_{H}-1\right\}$, the conventional generator $G_{k}$ is paid according to the locational marginal price $p_{k}\left(q_{k}(t)+\hat{w}_{k}(t)+r_{k}(t)\right)$. We define the profit of $G_{k}$ at $t$

\footnotetext{
${ }^{1}$ Other approaches exist: in lots of markets, exchanges between local markets come from generators within a real-time market (US), or is separately contracted (EU).

${ }^{2} \mathrm{By}$ comparison, the EU system relies on zonal pricing, i.e., there is a unique market price per zone [32]. The delimitation of the zones, which may contain multiple nodes, is a difficult problem.
} 
as the difference between the revenue generated by the selling of $q_{k}(t)$ units at market price $p_{k}(t)$ and the cost of these units production:

$$
\pi_{G_{k}}\left(q_{k}(t), \boldsymbol{q}_{-\boldsymbol{k}}(\boldsymbol{t}), \boldsymbol{r}(\boldsymbol{t}), \hat{\boldsymbol{w}}(\boldsymbol{t})\right)=q_{k}(t) p_{k}\left(q_{k}(t)+\hat{w}_{k}(t)+r_{k}(t)\right)-c_{k}\left(q_{k}(t)\right) .
$$

Assuming a zero marginal cost for the production of renewable energy, the profit of the renewable producer in local market $k$ at time period $t$ is defined as the revenue generated from the selling of $\hat{w}_{k}(t)$ at market price $p_{k}(t)$ :

$$
\tilde{\pi_{k}}\left(q_{k}(t), \boldsymbol{q}-\boldsymbol{k}(\boldsymbol{t}), \boldsymbol{r}(\boldsymbol{t}), \hat{\boldsymbol{w}}(\boldsymbol{t})\right)=\hat{w}_{k}(t) p_{k}\left(q_{k}(t)+\hat{w}_{k}(t)+r_{k}(t)\right) .
$$

The national MO profit function is the following:

$$
\pi_{M O}(\boldsymbol{q}(\boldsymbol{t}), \boldsymbol{r}(\boldsymbol{t}), \hat{\boldsymbol{w}}(\boldsymbol{t}))=\sum_{k=1, \ldots, n} r_{k}(t) p_{k}\left(q_{k}(t)+\hat{w}_{k}(t)+r_{k}(t)\right) .
$$

The national MO's objective coincides with social welfare maximization, which is defined as the maximization of the sum of the surplus of consumers and generators (here, conventional and renewable generators) and the national MO's profit:

$$
\begin{aligned}
& \mathcal{S W}(\boldsymbol{q}(\boldsymbol{t}), \boldsymbol{r}(\boldsymbol{t}), \hat{\boldsymbol{w}}(\boldsymbol{t})) \\
= & \sum_{k=1}^{n}\{\underbrace{\left(\int_{0}^{q_{k}(t)+\hat{w}_{k}(t)+r_{k}(t)}\left(p_{k}(u) d u-p_{k}\left(q_{k}(t)+\hat{w}_{k}(t)+r_{k}(t)\right)\right) d u\right.}_{\text {local market } k \text { consumer surplus }} \\
+ & \left.\hat{w}_{k}(t) p_{k}\left(q_{k}(t)+\hat{w}_{k}(t)+r_{k}(t)\right)+q_{k}(t) p_{k}\left(q_{k}(t)+w_{k}(t)+r_{k}(t)\right)-c_{k}\left(q_{k}(t)\right)\right\} \\
+ & \sum_{k=1, \ldots, n} r_{k}(t) p_{k}\left(q_{k}(t)+\hat{w}_{k}(t)+r_{k}(t)\right), \\
= & \sum_{k=1}^{n}\left\{\int_{0}^{q_{k}(t)+\hat{w}_{k}(t)+r_{k}(t)} p_{k}(u) d u-c_{k}\left(q_{k}(t)\right)\right\} .
\end{aligned}
$$

\subsection{Solution concepts}

The goal of this section is to formally introduce game theoretical solution concepts that will be used throughout the article. We start by introducing the notion of strategy and strategy profile.

Definition 2. [25] The strategy set of generators $G_{k}, k \in\{1, \ldots, n\}$, is the set of actions (block bids) $\boldsymbol{q}_{\boldsymbol{k}} \in \mathbb{R}^{t_{H}}$ that check the constraints defining the generator's feasible set. Similarly, the strategy set of the national MO is the set of exchanges $(\boldsymbol{r}(\boldsymbol{t}))_{t=0}^{t_{H}-1} \in$ $\mathcal{X}^{t_{H}}$ that check the demand and generation balance equation.

The first solution concept that we define ignores the sequential structure of the game; it treats the strategies as choices that are made once and for all before play begins. We assume that the exchanges between the local markets, $\boldsymbol{r}$, is fixed.

Definition 3. [25] A Nash equilibrium (for the Nash-Cournot game occurring between the generators) is a strategy profile $\left(\boldsymbol{q}^{\star}(\boldsymbol{t})\right)_{t=0}^{t_{H}-1}$ such that for every generator $G_{k}, k \in$ 


$$
\begin{aligned}
& \{1, \ldots, n\} \\
& \quad \sum_{t=0}^{t_{H}-1} \pi_{G_{k}}\left(q_{k}^{\star}(t), \boldsymbol{q}_{-\boldsymbol{k}}^{\star}(\boldsymbol{t}), \boldsymbol{r}(\boldsymbol{t}), \hat{\boldsymbol{w}}(\boldsymbol{t})\right) \geq \sum_{t=0}^{t_{H}-1} \pi_{G_{k}}\left(q_{k}(t), \boldsymbol{q}_{-\boldsymbol{k}}^{\star}(\boldsymbol{t}), \boldsymbol{r}(\boldsymbol{t}), \hat{\boldsymbol{w}}(\boldsymbol{t})\right),
\end{aligned}
$$

for all $\boldsymbol{q}_{\boldsymbol{k}}$ in generator $G_{k}$ 's strategy set.

Stackelberg games are two-stage games involving multiple leaders (generators) and a follower (the national MO). In the literature, they are often assimilated with EPEC [12]. Two-stage games can be modeled as extensive form games ${ }^{3}$. A subgame is part of this extensive form game that constitutes a valid extensive form game on its own. In a perfect information extensive form game, every action of the agents (generators or national MO) initializes a subgame [25]. This enables us to introduce the definition of a subgame perfect Nash equilibrium.

Definition 4. [25] A subgame perfect Nash equilibrium is a strategy profile $\left(\boldsymbol{q}^{\star}, \boldsymbol{r}^{\star}\right)$ for which for any history $h$ (i.e., generators' actions, or sequence of actions of generators and national $M O)$ the strategy profile $\left.\left(\boldsymbol{q}^{\star}, \boldsymbol{r}^{\star}\right)\right|_{h}$ is a Nash equilibrium of the subgame induced by $h$.

Since the whole game is a subgame, every subgame perfect Nash equilibrium is a Nash equilibrium. Subgame perfect Nash equilibrium can be found using simple algorithm known as backward induction [31].

\section{Mathematical programming formulation}

The two market designs described in Section 1, can be modeled as mathematical programs that we detail in Subsection 3.1 (centralized market design) and 3.2 (decentralized market design). Proofs for the existence and uniqueness of market equilibrium for the decentralized market design are provided in Subsection 3.3. Finally, an analytical comparison of sequential versus simultaneous market clearing is provided in Subsection 3.4.

\subsection{A standard optimization problem for the centralized market}

With the centralized market design, we assume that the national MO simultaneously balances supply and demand by optimizing exchanges, $r_{k}(t)$, between local markets $k \in\{1, \ldots, n\}$ such that demand at node $k$ receives a quantity $d_{k}(t)=q_{k}(t)+\hat{w}_{k}(t)+$ $r_{k}(t)$, and optimizes the quantities produced by the conventional generators under nonnegativity and inter-temporal constraints. The centralized market is here modeled as a one-level standard optimization problem under constraints on the quantity bids (nonnegativity, inter-temporality) and $\boldsymbol{r}(\boldsymbol{t})$ belongs to the injection region $\mathcal{X}$ defined in

\footnotetext{
${ }^{3}$ An extensive form game describes with a tree how a game is played. It depicts the order in which players make moves, and the information each player has at each decision point [25].
} 
Equation (1) at any time period $t \in\left\{0, \ldots, t_{H}-1\right\}$ :

$$
\begin{aligned}
\max _{(\boldsymbol{q}(\boldsymbol{t}))_{t=0}^{t_{H}-1},(\boldsymbol{r}(\boldsymbol{t}))_{t=0}^{t_{H}-1}} & \sum_{t=0}^{t_{H}-1} \mathcal{S W}(\boldsymbol{q}(\boldsymbol{t}), \boldsymbol{r}(\boldsymbol{t}), \hat{\boldsymbol{w}}(\boldsymbol{t})), \\
\text { s.t. } & q_{k}(t) \geq 0, \forall t=0, \ldots, t_{H}-1, \forall k=1, \ldots, n, \\
& \Phi\left(\boldsymbol{q}_{\boldsymbol{k}}\right) \leq \mathbf{0}, \forall k=1, \ldots, n,
\end{aligned}
$$

where $\Phi: \mathbb{R}^{t_{H}} \rightarrow \mathbb{R}^{m}$ is continuous, bijective following Definition 1 and $m \in \mathbb{N}^{*}$ is the number of inter-temporal constraints that we consider for the block bids.

\subsection{A bilevel mathematical program for local markets}

With the decentralized market design, the national MO optimizes only the exchanges between the local markets to guarantee that the aggregated supply and demand balance in each local market. Conventional generators declare simultaneously and independently quantity offers (block bids) in the local market ${ }^{4}$. The local market is modeled as a Stackelberg game where generators $\left(G_{k}\right)_{k=1, \ldots, n}$ act as leaders with the national $\mathrm{MO}$ as a follower. It is therefore formulated as a bilevel mathematical programming problem, where generators bid in their local market to maximize their profits, subject to inter-temporal constraints on the block quantity offers, while anticipating the outcome of the national market clearing. Bilevel problems are challenging to solve because they are generally non-convex [7].

The variables are divided into two classes, namely the lower-level variables $\boldsymbol{r}(\boldsymbol{t}) \in$ $\mathcal{X}, \forall t \in\left\{0, \ldots, t_{H}-1\right\}$, which determine the exchanges between the local markets at time period $t$, and the upper-level variables $\boldsymbol{q}_{\boldsymbol{k}} \in \mathbb{R}^{t_{H}}, \forall k=1, \ldots, n$, which capture the sequences of simple bids made by the generators. Mathematical programs (8), (9), (10) and (11), (12) are connected through the use of common variables, namely the sequence of exchanges between the local markets over time horizon $t_{H},(\boldsymbol{r}(\boldsymbol{t}))_{t=0}^{t_{H}-1}$. Also, the profits of the generators in Equation (8) cannot be computed until the sequences of exchanges, $(\boldsymbol{r}(\boldsymbol{t}))_{t=0}^{t_{H}-1}$, between the local markets are known. These exchanges are not in the direct control of the generators, but the solution of a mathematical program parametrized in the generators' bids $(\boldsymbol{q}(\boldsymbol{t}))_{t=0}^{t_{H}-1}$.

Formally, for all $k=1, \ldots, n$, the bilevel optimization problem writes down as follows:

for all $k=1, \ldots, n$,

$$
\begin{aligned}
& \max _{\boldsymbol{q}_{\boldsymbol{k}} \in \mathbb{R}^{t} H,(\boldsymbol{r}(\boldsymbol{t}))_{t=0}^{t_{H}-1} \in \mathcal{X}^{t} H} \sum_{t=0}^{t_{H}-1} \pi_{G_{k}}\left(q_{k}(t), \boldsymbol{q}_{-\boldsymbol{k}}(\boldsymbol{t}), \boldsymbol{r}(\boldsymbol{t}), \hat{\boldsymbol{w}}(\boldsymbol{t})\right), \\
& \text { s.t. } \quad q_{k}(t) \geq 0, \forall t \in\left\{0, \ldots, t_{H}-1\right\}, \\
& \Phi\left(\boldsymbol{q}_{\boldsymbol{k}}\right) \leq \mathbf{0} .
\end{aligned}
$$

${ }^{4}$ The national MOs are responsible for the clearing of their local markets. It can happens either simultaneously or sequentially. 
for all $t=0, \ldots, t_{H}-1$,

$$
\begin{array}{rl}
\boldsymbol{r}(\boldsymbol{t})=\arg \max _{\boldsymbol{r}^{\prime}(\boldsymbol{t}) \in \mathcal{X}} & \mathcal{S W}\left(\boldsymbol{q}(\boldsymbol{t}), \boldsymbol{r}^{\prime}(\boldsymbol{t}), \hat{\boldsymbol{w}}(\boldsymbol{t})\right), \\
\text { s.t. } & \sum_{k=1}^{n} r_{k}(t)=0 .
\end{array}
$$

As classical in bilevel optimization [6, 7], the lower-level problem in Equations (11) and (12) is nested within the upper-level problem described by Equations (8)-(10). The lower-level problem describes a setting where the national MO optimizes consumption only, and is strongly related to Yao et al. [36] formulation of the Cournot problem, but differs from it because their Cournot players cannot affect the price difference between buses.

At the upper-level, Equation (9) imposes non-negativity of the bid quantity submitted by $G_{k}$ in local market node $k$ and Equation (10) captures the inter-temporality constraints associated with bid $\boldsymbol{q}_{\boldsymbol{k}}$. At the lower-level, Equation (12) captures the fact that the sum of the exchanges between local markets, $\boldsymbol{r}(\boldsymbol{t}) \in \mathcal{X}$, vanishes at each time period $t \in\left\{0, \ldots, t_{H}-1\right\}$, by definition of the set $\mathcal{X}$ introduced in Equation (1). Equation (12) can easily be reformulated as an equation which represents demand and supply equilibrium, i.e., $\sum_{k=1}^{n} q_{k}(t)+\sum_{k=1}^{n} \hat{w}_{k}(t)=\sum_{k=1}^{n} d_{k}(t), \forall t \in\left\{0, \ldots, t_{H}-1\right\}$.

Furthermore, note that there is a temporal scale difference between the upper and the lower level: while $G_{k}$ defines block bids over time period $\left\{0, \ldots, t_{H}-1\right\}$, the national MO re-optimizes the exchanges between local markets at each time period $t \in\left\{0, \ldots, t_{H}-1\right\}$.

\subsection{Solving the bilevel mathematical program}

The inverse demand function $p_{k}: \mathbb{R}_{+} \rightarrow \mathbb{R}$ is linear (a fortiori concave) and strictly decreasing in $q_{k}$. Hence $\left.\left.p_{k}^{-1}:\right]-\infty ; a_{k}\right] \rightarrow \mathbb{R}_{+}$is well-defined and shares the same properties as $p_{k}$. We introduce the function $\left.\left.D:\right]-\infty, \max _{k=1, \ldots, n} a_{k}\right] \rightarrow \mathbb{R}_{+}$such that:

$$
D(x):=\sum_{k=1}^{n} p_{k}^{-1}(x) .
$$

For $D$ to be well-defined and striclty decreasing and concave (as the sum of such functions), we make the assumption that the price intercepts are identical i.e., $a_{k}=$ $a, \forall k=1, \ldots, n$. Under this assumption, we can properly introduce the inverse demand function $\left.\left.D^{-1}: \mathbb{R}_{+} \rightarrow\right]-\infty ; a\right]$ which is strictly decreasing and concave.

Proposition 1. For simple bids, the equilibrium $\left(\boldsymbol{q}^{\star}(\hat{\boldsymbol{w}}), \boldsymbol{r}^{\star}(\hat{\boldsymbol{w}})\right)$ solution of the Stackelberg game described by Equations (8), (9), (10), (11), (12) coincides with the social welfare optimum of a Cournot game between the $n$ conventional generators.

Proof of Proposition 1. For the sake of simplicity in this proof we omit the $t$ index since for simple bids we consider solely one time period. We start by computing the reaction function $\rho($.$) solution of the lower-level optimization problem that checks$ demand and supply balance, i.e.:

$$
\boldsymbol{d}(\boldsymbol{q}, \hat{\boldsymbol{w}})=\boldsymbol{q}+\hat{\boldsymbol{w}}+\underbrace{\rho(\boldsymbol{q})}_{r} .
$$


Making change of variable in the lower-level problem, i.e., replacing the decision variable $\boldsymbol{r}$ by $\boldsymbol{d}$, Equations (11) and (12) can be re-written:

$$
\begin{array}{ll}
\max _{\boldsymbol{d} \in \mathbb{R}_{+}^{n}} & \sum_{k=1}^{n}\left\{\int_{0}^{d_{k}} p_{k}(u) d u-c_{k}\left(q_{k}\right)\right\}, \\
\text { s.t. } & \sum_{k=1}^{n} d_{k}=\sum_{k=1}^{n} q_{k}+\sum_{k=1}^{n} \hat{w}_{k} .
\end{array}
$$

Note that $\int_{0}^{d_{k}} p_{k}(u) d u=\int_{0}^{d_{k}}\left(a-b_{k} u\right) d u=\left(a-\frac{b_{k}}{2} d_{k}\right) d_{k}$. Hence the objective function in Equation (15) is strictly concave. This implies that the lower-level equivalent problem described by Equations (15) and (16) admits a unique solution $\boldsymbol{d}^{\star}(\boldsymbol{q}, \hat{\boldsymbol{w}})$. In turn, this implies that there exists a unique reaction function $\rho^{\star}($.$) solution of$ Equation (14).

We now introduce the Lagrangian function associated with Equations (15) and (16):

$$
L(\boldsymbol{d}, \lambda)=\sum_{k=1}^{n}\left\{\int_{0}^{d_{k}} p_{k}(u) d u-c_{k}\left(q_{k}\right)\right\}+\lambda\left(\sum_{k=1}^{n} d_{k}-\sum_{k=1}^{n} q_{k}-\sum_{k=1}^{n} \hat{w}_{k}\right),
$$

where $\lambda \in \mathbb{R}$ is the Lagrange multiplier associated with lower-level constraint (12).

Differentiating $L(d, \lambda)$ with respect to $d_{k}$, we obtain:

$$
\begin{aligned}
\frac{\partial L(\boldsymbol{d}, \lambda)}{\partial d_{k}}=0 & \Leftrightarrow \lambda=p_{k}\left(d_{k}\right), \\
& \Leftrightarrow p_{k}^{-1}(\lambda)=d_{k} .
\end{aligned}
$$

By summation over $k=1, \ldots, n$ and definition of the market demand introduced in Equation (13), we obtain: $D(\lambda)=\sum_{k=1}^{n} d_{k}$. Using Equation (16), it can be rewritten: $D(\lambda)=\sum_{k=1}^{n} q_{k}+\sum_{k=1}^{n} \hat{w}_{k}$. From which we infer:

$$
\lambda=D^{-1}\left(\sum_{k=1}^{n} q_{k}+\sum_{k=1}^{n} \hat{w}_{k}\right)
$$

By substitution of Equation (18) in Equation (17), we obtain:

$$
d_{k}^{\star}(\boldsymbol{q}, \hat{\boldsymbol{w}})=p_{k}^{-1}\left(D^{-1}\left(\mathbf{1}^{T} \boldsymbol{q}+\mathbf{1}^{T} \hat{\boldsymbol{w}}\right)\right) .
$$

From which we infer the reaction function closed form expression: $\rho^{\star}(\boldsymbol{q}, \hat{\boldsymbol{w}})=$ $d^{\star}(\boldsymbol{q}, \hat{\boldsymbol{w}})-\boldsymbol{q}-\hat{\boldsymbol{w}}$.

The space of definition of $\boldsymbol{r}$ in the lower-level problem (11)-(12) imposes that:

$$
\rho^{\star}(\boldsymbol{q}, \hat{\boldsymbol{w}}) \in \mathcal{X} \Leftrightarrow \sum_{k=1}^{n} \rho^{\star}\left(q_{k}, \hat{\boldsymbol{w}}\right)=0,-\boldsymbol{f} \leq \boldsymbol{H} \rho^{\star}(\boldsymbol{q}, \hat{\boldsymbol{w}}) \leq \boldsymbol{f}
$$

This means that for a solution $\left(\boldsymbol{q}, \rho^{\star}(\boldsymbol{q}, \hat{\boldsymbol{w}})\right)$ to be feasible in the upper-level problem, the generators need to select $\boldsymbol{q}$ that check (9), (10) and $-\boldsymbol{f} \leq \boldsymbol{H} \rho^{\star}(\boldsymbol{q}, \hat{\boldsymbol{w}}) \leq \boldsymbol{f}$, the constraint $\sum_{k=1}^{n} \rho^{\star}\left(q_{k}, \hat{\boldsymbol{w}}\right)=0$ being automatically checked through Equation (16). In practice, we will assume that a generator will always presumes that the transmission network constraints remain non-binding (e.g., they are not saturated) when calculating 
the subgame perfect Nash equilibrium output ${ }^{5}$. If the anticipated constraints coincide with the realized constraints then the belief of the generators is consistent, and the algorithm converges to an equilibrium; otherwise oscillations might appear [23].

We now substitute the reaction function $\rho^{\star}($.) in the upper-level optimization problem objective function:

$$
\begin{aligned}
\pi_{G_{k}}\left(q_{k}, \boldsymbol{q}-\boldsymbol{k}, \rho^{\star}(\boldsymbol{q}, \hat{\boldsymbol{w}}), \hat{\boldsymbol{w}}\right) & =p_{k}\left(d_{k}^{\star}(\boldsymbol{q}, \hat{\boldsymbol{w}})\right) q_{k}-c_{k}\left(q_{k}\right), \\
& =D^{-1}\left(\mathbf{1}^{T} \boldsymbol{q}_{-\boldsymbol{k}}+q_{k}+\mathbf{1}^{T} \hat{\boldsymbol{w}}\right) q_{k}-c_{k}\left(q_{k}\right)
\end{aligned}
$$

using Equation (19). We observe that Equation (20) becomes independent of the lower-level reaction function $\rho^{\star}(\boldsymbol{q}, \hat{\boldsymbol{w}})$. As a result, an equilibrium of the Stackelberg game (a subgame perfect Nash equilibrium following Definition 4) is also an equilibrium of a corresponding Cournot game involving the $n$ conventional generators and $D^{-1}\left(\mathbf{1}^{T} \boldsymbol{q}-\boldsymbol{k}+q_{k}+\mathbf{1}^{T} \hat{\boldsymbol{w}}\right)$ as inverse demand function.

An important by-product of this result is that there is no difference between local market prices. Also, note that in case where the cost function of the generators is quadratic (i.e., $c_{k}\left(q_{k}\right)=c_{k} q_{k}^{2}, c_{k}>0, \forall k \in\{1, \ldots, n\}$ ), each conventional generator which is producing more energy makes the profit of all the other conventional generators lower since the market price is decreasing as a function of the total production.

We now introduce an intermediate result on the generators' profit functions.

Lemma 1. The generator $G_{k}$ 's profit function $\pi_{G_{k}}\left(q_{k}, \boldsymbol{q}-\boldsymbol{k}, \rho^{\star}(\boldsymbol{q}, \hat{\boldsymbol{w}}), \hat{\boldsymbol{w}}\right)$ is twice continuously differentiable and strictly concave with respect to own output $q_{k}$.

Proof of Lemma 1. For the sake of simplicity we set $Q:=\mathbf{1}^{T} \boldsymbol{q}_{-\boldsymbol{k}}+q_{k}+\mathbf{1}^{T} \hat{\boldsymbol{w}}$. We mentioned above that the inverse market demand function $D^{-1}($.$) is concave$ and strictly decreasing with respect to $q_{k}$ which implies that $\frac{\partial^{2} D^{-1}(Q)}{\partial q_{k}^{2}}<0$ and $\frac{\partial D^{-1}(Q)}{\partial q_{k}}<0$ respectively. Differentiating twice $\pi_{G_{k}}$ with respect to $q_{k}$, we obtain: $\frac{\partial^{2} \pi_{G_{k}}\left(q_{k}, \boldsymbol{q}_{-\boldsymbol{k}}, \rho^{\star}(\boldsymbol{q}), \hat{\boldsymbol{w}}\right)}{\partial q_{k}^{2}}=q_{k} \underbrace{\frac{\partial^{2} D^{-1}(Q)}{\partial q_{k}^{2}}}_{<0}+2 \underbrace{\frac{\partial D^{-1}(Q)}{\partial q_{k}}}_{<0}-\underbrace{\frac{\partial^{2} c_{k}\left(q_{k}\right)}{\partial q_{k}^{2}}}_{>0}<0$, which implies that $\pi_{G_{k}}($.$) is striclty concave with respect to q_{k}$.

Proposition 2. For simple bids, we prove that the Stackelberg game described by Equations (8), (9), (10), (11), (12) is equivalent to a non linear Complementarity Problem.

Proof of Proposition 2. Considering simple bids means that there is no intertemporal constraints, i.e., $\Phi(.) \equiv 0$. It is well-known that the set of Cournot-Nash equilibria is the same as the set of simultaneous solutions of the first-order optimality

\footnotetext{
${ }^{5}$ The case where generators anticipate transmission link congestion is for example addressed in [19].
} 
conditions of the $n$ individual generators' problems [34]:

$$
\begin{aligned}
\Psi_{k}(\boldsymbol{q}, \hat{\boldsymbol{w}}):= & \frac{\partial \pi_{G_{k}}\left(q_{k}, \boldsymbol{q}-\boldsymbol{k}, \rho^{\star}(\boldsymbol{q}, \hat{\boldsymbol{w}}), \hat{\boldsymbol{w}}\right)}{\partial q_{k}} \\
= & \frac{\partial D^{-1}(Q)}{\partial q_{k}} q_{k}+D^{-1}(Q)-c_{k}^{\prime}\left(q_{k}\right) \geq 0 \\
& q_{k} \frac{\partial \pi_{G_{k}}\left(q_{k}, \boldsymbol{q}-\boldsymbol{k}, \rho^{\star}(\boldsymbol{q}, \hat{\boldsymbol{w}}), \hat{\boldsymbol{w}}\right)}{\partial q_{k}}=0, \\
& q_{k} \geq 0 .
\end{aligned}
$$

This system of equations can be reformulated as a non linear Complementarity Problem $C P\left(\mathbb{R}_{+}^{n}, \boldsymbol{\Psi}\right)$ :

$$
\begin{aligned}
& \boldsymbol{\Psi}(\boldsymbol{q}, \hat{\boldsymbol{w}}) \geq 0 \\
& \boldsymbol{q}^{T} \boldsymbol{\Psi}(\boldsymbol{q}, \hat{\boldsymbol{w}})=0 \\
& \boldsymbol{q} \geq 0
\end{aligned}
$$

where we set $\boldsymbol{\Psi}(\boldsymbol{q}, \hat{\boldsymbol{w}}):=\left(\Psi_{1}(\boldsymbol{q}, \hat{\boldsymbol{w}}), \ldots, \Psi_{n}(\boldsymbol{q}, \hat{\boldsymbol{w}})\right)$. The generators' objective functions $\pi_{G_{k}}\left(q_{k}, \boldsymbol{q}-\boldsymbol{k}, \rho^{\star}(\boldsymbol{q}, \hat{\boldsymbol{w}}), \hat{\boldsymbol{w}}\right)$ being twice continuously differentiable and striclty concave with respect to $q_{k}$ for all $k \in\{1, \ldots, n\}$ according to Lemma 1, Kolstad and Mathiesen proved in [15] that finding a Nash-Cournot equilibrium $\boldsymbol{q}^{\star}$ is equivalent to find $\boldsymbol{q}^{\star} \in C P\left(\mathbb{R}_{+}^{n}, \boldsymbol{\Psi}\right)$.

We now try to generalize this result in case where inter-temporal constraints hold, i.e., $\Phi(.) \not \equiv 0$. Let introduce the set

$$
\mathcal{K}:=\left\{\boldsymbol{q} \in \mathbb{R}_{+}^{n} \mid \Phi\left(q_{k}\right) \leq \mathbf{0}, \forall k \in\{1, \ldots, n\}\right\}
$$

$\mathcal{K}$ is a subset of $\mathbb{R}^{n}$. First, we check that $\left.\left.\mathcal{K}=\Phi^{-1}(]-\infty ; 0\right]^{m}\right)$ is closed as reciprocal image of a closed subset of $\mathbb{R}^{m}$ through a continuous application. By definition, $\mathcal{K}$ is a bounded subset of $\mathbb{R}^{n}$. Since compacts in $\mathbb{R}^{n}$ are in general the closed and bounded subsets, we have proved that $\mathcal{K}$ is compact in $\mathbb{R}_{+}^{n}$.

We now prove some properties of the $\boldsymbol{\Psi}$ vector.

Lemma 2. $\nabla \boldsymbol{\Psi}$ is an Hadamard matrix if, and only if

$$
c_{k}^{\prime \prime}\left(q_{k}\right)>(n-3) \frac{1}{\sum_{l=1}^{n} \frac{1}{b_{l}}}, \forall k \in\{1, \ldots, n\} .
$$

Proof of Lemma 2. By definition $p_{k}(Q)=a_{k}-b_{k} Q \Leftrightarrow Q=\frac{a_{k}}{b_{k}}-\frac{1}{b_{k}} p_{k}(Q), \forall k \in$ $\{1, \ldots, n\}$. Substituting $p_{k}^{-1}(Q)$ in $Q$ we obtain $p_{k}^{-1}(Q)=\frac{a_{k}}{b_{k}}-\frac{1}{b_{k}} Q$. Then using the definition of the demand function $D(Q)=\sum_{k=1}^{n} p_{k}^{-1}(Q)$, we infer that $D(Q)=$ $\left(\sum_{k=1}^{n} \frac{a_{k}}{b_{k}}\right)-\left(\sum_{k=1}^{n} \frac{1}{b_{k}}\right) Q$. Using the same principle, we infer that:

$$
D^{-1}(Q)=\underbrace{\frac{\sum_{k=1}^{n} \frac{a_{k}}{b_{k}}}{\sum_{k=1}^{n} \frac{1}{b_{k}}}}_{A}-\underbrace{\frac{1}{\sum_{k=1}^{n} \frac{1}{b_{k}}}}_{B} Q .
$$


Differentiating $D^{-1}(Q)$ with respect to $q_{k}$, we obtain $\frac{\partial D^{-1}(Q)}{\partial q_{k}}=-B, \frac{\partial^{2} D^{-1}(Q)}{\partial q_{k}^{2}}=$ $0, \forall k \in\{1, \ldots, n\}$. For $\nabla \boldsymbol{\Psi}$ to be an Hadamard matrix we need to check the row strictly diagonally dominance condition, i.e.,

$$
\begin{array}{r}
\left|\frac{\partial^{2}}{\partial q_{k}^{2}} D^{-1}(Q) q_{k}+2 \frac{\partial}{\partial q_{k}} D^{-1}(Q)-c_{k}^{\prime \prime}\left(q_{k}\right)\right|>\sum_{l=1, l \neq k}^{n}\left|\frac{\partial^{2}}{\partial q_{l} \partial q_{k}} D^{-1}(Q) q_{k}+\frac{\partial}{\partial q_{l}} D^{-1}(Q)\right| \\
\Leftrightarrow 2 B+c_{k}^{\prime \prime}\left(q_{k}\right)>(n-1) B \\
\Leftrightarrow c_{k}^{\prime \prime}\left(q_{k}\right)>(n-3) B .
\end{array}
$$

We now come to the more general result below

Proposition 3. The Stackelberg game described by Equations (8), (9), (10), (11), (12) admits a unique equilibrium provided Equation (22) holds.

Proof of Proposition 3. We checked in Lemma 1 that each generator $G_{k}$ profit function is twice differentiable and striclty concave with respect to $q_{k}$, and in Lemma 2 that $\nabla \boldsymbol{\Psi}$ is an Hadamard matrix if, and only if, Equation (22) is checked. Then following Moré and Rheinbold in [22], there exists at most one solution to $C P\left(\mathbb{R}_{+}^{n}, \Psi\right)$. This would prove the uniqueness of $\boldsymbol{q}^{\star}$. We now deal with existence.

Let $\boldsymbol{q} \in \mathbb{R}_{+}^{n} \backslash \mathcal{K}$, then there exist at least one $k \in\{1, \ldots, n\}$ such that $\Phi\left(q_{k}\right)>0 \Leftrightarrow$ $q_{k}>\Phi^{-1}(0)$ if $\Phi^{-1}$ is monotonically increasing. We build a vector $\boldsymbol{y} \in \mathbb{R}_{+}^{n}$ such that $y_{k}<\Phi^{-1}(0)$ for all $k \in\{1, \ldots, n\}$ such that $q_{k}>\Phi^{-1}(0)$ and for the remaining $k$, choose $y_{k}=q_{k}$. Then $(\boldsymbol{q}-\boldsymbol{y})^{T} \boldsymbol{\Psi}(\boldsymbol{q}, \hat{\boldsymbol{w}})=\sum_{\left\{k \in\{1, \ldots, n\} \mid q_{k}>\Phi^{-1}(0)\right\}}\left(q_{k}-y_{k}\right) \frac{\partial \pi_{G_{k}}(\boldsymbol{q}, \hat{\boldsymbol{w}})}{\partial q_{k}}>0$ by definition of $\boldsymbol{q}$ and construction of $\boldsymbol{y}$. The same construction can be transposed to the case where $\Phi$ is monotonically decreasing. In that case, for all the $k \in\{1, \ldots, n\}$ such that $\Phi\left(q_{k}\right)>0$, we choose $y_{k}=q_{k}$, and for the remaining ones, we choose $y_{k}<\Phi^{-1}(0)$.

Following [14] this proves that if Equation (22) is checked, there exists a unique Cournot-Nash equilibrium for the non-cooperative game between the generators, that we call $\boldsymbol{q}^{\star}(\hat{\boldsymbol{w}})$.

The precedent steps lead us to the conclusion that there exists a unique equilibrium $\left(\boldsymbol{q}^{\star}(\hat{\boldsymbol{w}}), \rho^{\star}\left(\boldsymbol{q}^{\star}, \hat{\boldsymbol{w}}\right)\right)$ solution of the Stackelberg game described by Equations (8), (9), (10), (11), (12).

Proposition 4. For block bids, there exists a unique equilibrium $\left(\left(\boldsymbol{q}^{\star}(\hat{\boldsymbol{w}}(\boldsymbol{t}), \boldsymbol{t})\right)_{t=0}^{t_{H}-1},\left(\boldsymbol{r}^{\star}(\hat{\boldsymbol{w}}(\boldsymbol{t}), \boldsymbol{t})\right)_{t=0}^{t_{H}-1}\right)$ solution of the Stackelberg game, provided Equation (22) is checked at each time period $t \in\left\{0, \ldots, t_{H}-1\right\}$.

Proof of Proposition 4. We proceed by extension of Proposition 1 proof. The continuity and strict concavity of $\sum_{t=0}^{t_{H}-1} \pi_{G_{k}}\left(q_{k}(t), \boldsymbol{q}-\boldsymbol{k}(\boldsymbol{t}), \boldsymbol{r}(\boldsymbol{t}), \hat{\boldsymbol{w}}(\boldsymbol{t})\right)$ is straightforward as the sum of functions having this property. In Proposition 1 proof, the case where there is no inter-temporal constraint is straightforward to extend to block bids since the upper-level problem in Equations (8), (9) can be decomposed in independent problems over the temporal space $0, \ldots, t_{H}-1$. In case where inter-temporal constraints are introduced, Lemma 2 can be extended to prove that $\nabla \boldsymbol{\Psi}(\boldsymbol{q}(\boldsymbol{t}), \hat{\boldsymbol{w}}(\boldsymbol{t}))$ is an Hadamard matrix at any time period $t \in\left\{0, \ldots, t_{H}-1\right\}$. The end of the proof is straightforward to transpose to block bids by observing that $\mathcal{K}^{n}:=\left\{\left(\boldsymbol{q}_{\boldsymbol{k}}\right)_{k=1}^{n} \in \mathbb{R}_{+}^{n t_{H}} \mid \Phi\left(\boldsymbol{q}_{\boldsymbol{k}}\right) \leq \mathbf{0}, \forall k \in\right.$ 
$\left.\left.\{1, \ldots, n\}\}=\prod_{k=1, \ldots, n} \Phi^{-1}(]-\infty ; 0\right]^{m}\right)$ is closed as the product of closed sets, and that $(\boldsymbol{q}-\boldsymbol{y})^{T} \boldsymbol{\Psi}(\boldsymbol{q}, \hat{\boldsymbol{w}})=\sum_{t=0}^{t_{H}-1}(\boldsymbol{q}(\boldsymbol{t})-\boldsymbol{y}(\boldsymbol{t}))^{T} \boldsymbol{\Psi}(\boldsymbol{q}(\boldsymbol{t}), \hat{\boldsymbol{w}}(\boldsymbol{t}))$. Then, following the same procedure as above, it is possible to construct vectors $\boldsymbol{y}(\boldsymbol{t}) \in \mathbb{R}_{+}^{n}$ such that $(\boldsymbol{q}(\boldsymbol{t})-\boldsymbol{y}(\boldsymbol{t}))^{T} \boldsymbol{\Psi}(\boldsymbol{q}(\boldsymbol{t}), \hat{\boldsymbol{w}}(\boldsymbol{t}))>0, \forall t \in\left\{0, \ldots, t_{H}-1\right\}$.

We have proved that the Stackelberg game between $n$ generators located in local markets and a national MO admits a unique equilibrium under mild assumptions.

\subsection{Sequential local market clearing}

In the previous section, the conventional generators optimize their quantity bids simultaneously and independently, and the local markets are cleared simultaneously by the national MO. Another market design can be envisaged in which the local markets are cleared sequentially by the national MO. Without loss of generalities, we assume that local market $k$ has lower priority than local market $k+1$ for any $k=1, \ldots, n-1$, in the sequential clearing, and that the cost functions are quadratic in the quantity produced, leading to $c_{k}\left(q_{k}\right)=c_{k} q_{k}^{2}, \forall k=1, \ldots, n$.

To solve the local market optimization sequentially, we have to decompose the upper-level problem in $n$ sequential optimization problems that will be solved proceeding by backward induction, i.e., starting with the lowest priority local market. The lower-level problem remains the same and the reaction function $\rho^{\star}(\boldsymbol{q})$ coincides with the one obtained in case of simultaneous local market clearing. We checked in Lemma 2 proof that it is possible to write the inverse demand function as $D^{-1}(Q)=A-B Q$ where $A:=\frac{\sum_{k=1}^{n} \frac{a_{k}}{b_{k}}}{\sum_{k=1}^{n} \frac{1}{b_{k}}}$ and $B:=\frac{1}{\sum_{k=1}^{n} \frac{1}{b_{k}}}$, assuming that there exists at least one $k \in\{1, \ldots, n\}$ such that $b_{k}>0$. Differentiating the conventional generator $G_{1}$ (lowest priority local market) profit function with respect to $q_{1}$ we obtain:

$$
\begin{aligned}
& \frac{\partial \pi_{G_{1}}\left(q_{1}, \boldsymbol{q}-\mathbf{1}, \rho^{\star}(\boldsymbol{q}), \hat{\boldsymbol{w}}\right)}{\partial q_{1}}=A-B\left(q_{1}+Q\right)-2 c_{1} q_{1}=0, \\
\Leftrightarrow \quad & A-B Q=\left(B+2 c_{1}\right) q_{1} .
\end{aligned}
$$

For any $k=2, \ldots, n$, by substitution of Equation (23) in any $G_{k}$ profit function, we obtain:

$$
\begin{aligned}
\pi_{G_{k}}\left(q_{k}, \boldsymbol{q}_{-\boldsymbol{k}}, \rho^{\star}(\boldsymbol{q}), \hat{\boldsymbol{w}}\right) & =(A-B Q) q_{k}-c_{k} q_{k}^{2}, \\
& =\left(B+2 c_{1}\right) q_{1} q_{k}-c_{k} q_{k}^{2} .
\end{aligned}
$$

Then differentiating $\pi_{G_{k}}$ with respect to $q_{k}$ we obtain:

$$
\begin{aligned}
& \frac{\partial \pi_{G_{k}}\left(q_{k}, \boldsymbol{q}_{-\boldsymbol{k}}, \rho^{\star}(\boldsymbol{q}), \hat{\boldsymbol{w}}\right)}{\partial q_{k}}=\left(B+2 c_{1}\right) q_{1}-2 c_{k} q_{k}=0, \\
\Leftrightarrow & \frac{\left(B+2 c_{1}\right) q_{1}}{2 c_{k}}=q_{k}, \forall k=2, \ldots, n .
\end{aligned}
$$

Proposition 5. Assuming that all the local markets are cleared sequentially, the optimal quantity bidded by the generator in the lowest priority local market is smaller than the optimal bid obtained when all the local markets are cleared simultaneously. 
Proof of Proposition 5. Using Equation (24) we infer that $Q=q_{1}+\sum_{k=2}^{n} \frac{B+2 c_{1}}{2 c_{k}} q_{1}+$ $\sum_{k=1}^{n} \hat{w}_{k}$. By substitution in Equation (23), we infer that $\left(B+2 c_{1}\right) q_{1}=A-B\left(q_{1}+\right.$ $\left.\sum_{k=2}^{n} \frac{B+2 c_{1}}{2 c_{k}} q_{1}+\sum_{k=1}^{n} \hat{w}_{k}\right)$ which is equivalent with:

$$
\left(2 B+2 c_{1}+B\left(B+2 c_{1}\right) \sum_{k=2}^{n} \frac{1}{2 c_{k}}\right) q_{1}=A-B \sum_{k=1}^{n} \hat{w}_{k} .
$$

If local markets are cleared simultaneously we have $q_{k}=\frac{B+2 c_{1}}{B+2 c_{k}} q_{1}, \forall k=2, \ldots, n$. Hence $Q=\left(B+2 c_{1}\right) \sum_{k=2}^{n} \frac{1}{B+2 c_{k}} q_{1}+q_{1}+\sum_{k=1}^{n} \hat{w}_{k}$. Using the relation $\left(B+2 c_{1}\right) q_{1}=A-B Q$ we infer that:

$$
\left(2 B+2 c_{1}+B\left(B+2 c_{1}\right) \sum_{k=2}^{n} \frac{1}{B+2 c_{k}}\right) q_{1}=A-B \sum_{k=1}^{n} \hat{w}_{k} .
$$

Using the definition of $B$, we conclude that the left part of Equation (26) is smaller than the left part of Equation (25).

There is no straightforward generalization of Proposition 5 to local markets with higher priority than 1 , and related results could only be derived numerically.

Note that Proposition 5 does not imply a last-mover advantage [20] in general because $\pi_{G_{1}}\left(q_{1}^{\star}, \boldsymbol{q}_{-\mathbf{1}}^{\star}, \hat{\boldsymbol{w}}\right)=(1-B Q)\left(q_{1}^{\star}\right)^{2}-c_{1}\left(q_{1}^{\star}\right)^{2}=\underbrace{\left(B+c_{1}\right)}_{>0}\left(q_{1}^{\star}\right)^{2}$ using the fact

that $A-B Q=\left(B+2 c_{1}\right) q_{1}^{\star}$, which holds both under simultaneous and sequential local market clearing. Proposition 5 implying that the lowest priority generator $G_{1}$ 's optimal bid is lower under sequential market clearing than under simultaneous, $G_{1}$ 's profit function, $\pi_{G_{1}}\left(q_{1}^{\star}, \boldsymbol{q}_{-\mathbf{1}}^{\star}, \hat{\boldsymbol{w}}\right)$ recalled just above, is smaller under sequential market clearing than under simultaneous market clearing. However, in Subsection 4.3.2 case study, we check numerically that sequential market clearing gives rise to a higher social welfare (as defined in Equation (11)) than simultaneous market clearing. Based on social welfare definition in Equation (7), this implies that sequential local market clearing may be more profitable to other agents (generators and MOs in local markets with higher priority than 1 , renewable producers, consumers) than simultaneous local market clearing.

\section{Case study}

We consider two market nodes interconnected by a single transmission line. Each generator has a quadratic cost function, i.e., $c_{k}\left(q_{k}\right)=c_{k} q_{k}^{2}, \forall k=1,2$. Furthermore $c_{2}>c_{1}>0$ and $r_{1}=-r_{2}=r$.

\subsection{Quantifying efficiency}

The Price of Anarchy (PoA) is defined as the ratio between the optimal social welfare and that of the worst value of an equilibrium [16, 18, 24]:

$$
\operatorname{PoA}(\hat{\boldsymbol{w}}):=\frac{\max _{\boldsymbol{q} \in \mathbb{R}_{+}^{n t_{H}}, \boldsymbol{r} \in \mathcal{X}^{n t}{ }_{H}} \mathcal{S W}(\boldsymbol{q}, \boldsymbol{r}, \hat{\boldsymbol{w}})}{\mathcal{S W}\left(\boldsymbol{q}^{\star}, \rho\left(\boldsymbol{q}^{\star}\right), \hat{\boldsymbol{w}}\right)} .
$$


The PoA is a measure of the efficiency loss when introducing anticipatory behavior from the generators located in local markets, compared with the centralized market design (where no anticipatory behavior holds). By definition, the PoA is always larger than 1 and the efficiency loss is minimal when the PoA approaches 1 [18].

\subsection{Computing solutions for centralized and decentralized market designs}

In the proposed case study, the social welfare can be expressed analytically as:

$$
\mathcal{S W}(\boldsymbol{q}, \boldsymbol{r}, \hat{\boldsymbol{w}})=\sum_{k=1,2}\left\{a_{k}\left(q_{k}+r_{k}+\hat{w}_{k}\right)-\frac{b_{k}}{2}\left(q_{k}+r_{k}+\hat{w}_{k}\right)^{2}-c_{k} q_{k}^{2}\right\} .
$$

Using Equation (28), we derive closed form expressions of solutions for the centralized market optimization problem given in Subsection 3.1 and Stackelberg game decribed in Equations (8)-(12).

\subsubsection{Centralized market}

In the centralized market organization, the national MO determines simultaneously the quantities produced by the generators $\left(q_{k}^{c}\right)_{k=1,2}$ and the exchanges between nodes $\boldsymbol{r}^{c}$. Differentiating the social welfare function in Equation (28) with respect to each of these variables, we obtain a linear system of equations in $q_{1}^{c}, q_{2}^{c}, r^{c}$ which admits a unique solution:

$$
\begin{array}{lll}
\left.\frac{\partial \mathcal{S W}(\boldsymbol{q}, \boldsymbol{r}, \hat{\boldsymbol{w}})}{\partial q_{k}}\right|_{q_{k}=q_{k}^{c}}=0 & \Leftrightarrow & q_{k}^{c}=\frac{a_{k}-b_{k} r^{c}-b_{k} \hat{w}_{k}}{b_{k}+2 c_{k}}, \forall k=1,2, \\
\left.\frac{\partial \mathcal{S} \mathcal{W}(\boldsymbol{q}, \boldsymbol{r}, \hat{\boldsymbol{w}})}{\partial r}\right|_{r=r^{c}}=0 & \Leftrightarrow \quad r^{c}=\frac{a_{1}+a_{2}-b_{1} q_{1}^{c}-b_{1} \hat{w}_{1}-b_{2} q_{2}^{c}-b_{2} \hat{w}_{2}}{b_{1}+b_{2}} .
\end{array}
$$

This is a linear system of equations that can be rewritten matricially as follows:

$$
\left(\begin{array}{ccc}
b_{1}+2 c_{1} & 0 & b_{1} \\
0 & b_{2}+2 c_{2} & b_{2} \\
b_{1} & b_{2} & b_{1}+b_{2}
\end{array}\right)\left(\begin{array}{c}
q_{1}^{c} \\
q_{2}^{c} \\
r^{c}
\end{array}\right)=\left(\begin{array}{c}
a_{1}-b_{1} \hat{w}_{1} \\
a_{2}-b_{2} \hat{w}_{2} \\
\left(a_{1}+a_{2}\right)-b_{1} \hat{w}_{1}-b_{2} \hat{w}_{2}
\end{array}\right)
$$

Since the discriminant of the system matrix is positive $\left(2 b_{1} b_{2} c_{1}+2 b_{1} b_{2} c_{2}+4 b_{1} c_{1} c_{2}+\right.$ $\left.4 b_{2} c_{1} c_{2}>0\right)$ because of the assumptions made on the parameters, the system admits a unique solution that can be expressed analytically:

$$
\begin{aligned}
q_{1}^{c}(\hat{\boldsymbol{w}}) & =\frac{a_{1} b_{2} c_{2}-a_{2} b_{1} c_{2}-b_{1} b_{2} c_{2} \hat{w}_{1}+b_{1} b_{2} c_{2} \hat{w}_{2}}{b_{1} b_{2} c_{1}+b_{1} b_{2} c_{2}+2 b_{1} c_{1} c_{2}+2 b_{2} c_{1} c_{2}} \\
q_{2}^{c}(\hat{\boldsymbol{w}}) & =\frac{-a_{1} b_{2} c_{1}+a_{2} b_{1} c_{1}+b_{1} b_{2} c_{1} \hat{w}_{1}-b_{1} b_{2} c_{1} \hat{w}_{2}}{b_{1} b_{2} c_{1}+b_{1} b_{2} c_{2}+2 b_{1} c_{1} c_{2}+2 b_{2} c_{1} c_{2}} \\
r^{c}(\hat{\boldsymbol{w}}) & =\left\{a_{1} b_{2} c_{1}+2 a_{1} c_{1} c_{2}+a_{2} b_{1} c_{2}+2 a_{2} c_{1} c_{2}-b_{1} b_{2} c_{1} \hat{w}_{1}-b_{1} b_{2} c_{2} \hat{w}_{2}-2 b_{1} c_{1} c_{2} \hat{w}_{1}\right. \\
& \left.-2 b_{2} c_{1} c_{2} \hat{w}_{2}\right\}\left\{b_{1} b_{2} c_{1}+b_{1} b_{2} c_{2}+2 b_{1} c_{1} c_{2}+2 b_{2} c_{1} c_{2}\right\}^{-1} .
\end{aligned}
$$




\subsubsection{Local markets with simultaneous market clearing}

With the local market organization, a Stackelberg game (EPEC [12, 23, 36]) occurs between the conventional generators who anticipate the outcome of the market clearing process, and the national MO. The Stackelberg game is formalized as a bilevel optimization problem involving an upper-level optimization problem decribed by Equations (8), (9), (10) inter-related with a lower-level optimization problem described in Equations (11), (12).

In this Stackelberg game setting, we are looking for subgame perfect Nash equilibrium. To determine the lower-level reaction function, we proceed by backward induction, i.e., we start by solving the lower-level optimization problem (11), (12) assuming that the generators' quantities $q_{1}, q_{2}$ are fixed. Differentiating the lower-level objective function with respect to $r$, we obtain the analytical expression of the reaction function:

$$
\rho^{\star}(\boldsymbol{q}, \hat{\boldsymbol{w}})=\frac{a_{1}+a_{2}-b_{1} q_{1}-b_{1} \hat{w}_{1}-b_{2} q_{2}-b_{2} \hat{w}_{2}}{b_{1}+b_{2}} .
$$

By substitution of the reaction function in the upper-level equations (8), (9) and (10) and differentiation with respect to $q_{k}(k=1,2)$, we obtain:

$$
\begin{aligned}
q_{k}^{\star}(\hat{\boldsymbol{w}}) & =\left\{3 a_{k} b_{k} b_{l}^{2}+2 a_{k} b_{k} b_{l} c_{l}+4 a_{k} b_{l}^{3}+2 a_{k} b_{l}^{2} c_{l}-a_{l} b_{k}^{2} b_{l}-2 a_{l} b_{k}^{2} c_{l}-2 a_{l} b_{k} b_{l}^{2}\right. \\
& -2 a_{l} b_{k} b_{l} c_{l}-3 b_{k}^{2} b_{l}^{2} \hat{w}_{k}+b_{k}^{2} b_{l}^{2} \hat{w}_{l}-2 b_{k}^{2} b_{l} c_{l} \hat{w}_{k}+2 b_{k}^{2} b_{l} c_{l} \hat{w}_{l}-4 b_{k} b_{l}^{3} \hat{w}_{k} \\
& \left.+2 b_{k} b_{l}^{3} \hat{w}_{l}-2 b_{k} b_{l}^{2} c_{l} \hat{w}_{k}+2 b_{k} b_{l}^{2} c_{l} \hat{w}_{l}\right\}\left\{5 b_{k}^{2} b_{l}^{2}+4 b_{k}^{2} b_{l} c_{k}+4 b_{k}^{2} b_{l} c_{l}+4 b_{k}^{2} c_{k} c_{l}\right. \\
& \left.+8 b_{k} b_{l}^{3}+12 b_{k} b_{l}^{2} c_{k}+4 b_{k} b_{l}^{2} c_{l}+8 b_{k} b_{l} c_{k} c_{l}+8 b_{l}^{3} c_{k}+4 b_{l}^{2} c_{k} c_{l}\right\}^{-1}, k, l=1,2, k \neq l .
\end{aligned}
$$

\subsubsection{Local markets with sequential market clearing}

In this section, we assume instead that the local markets are cleared sequentially. The reaction function is identical with Equation (29), i.e., in the case where local markets are cleared simultaneously. The optimal bids of the generators located in the two local markets (market 1 having lower priority than market 2) are computed analytically:

$$
\begin{aligned}
q_{1}^{\star}\left(q_{2}, \hat{\boldsymbol{w}}\right) & =\frac{a_{1} b_{2}-a_{2} b_{1}+b_{1} b_{2} q_{2}-b_{1} b_{2} \hat{w}_{1}+b_{1} b_{2} \hat{w}_{2}}{2 b_{1} b_{2}+2 b_{1} c_{1}+2 b_{2} c_{1}}, \\
q_{2}^{\star}(\hat{\boldsymbol{w}}) & =\left\{a_{1} b_{1} b_{2}^{2}+2 a_{1} b_{1} b_{2} c_{1}+2 a_{1} b_{2}^{2} c_{1}+3 a_{2} b_{1}^{2} b_{2}+2 a_{2} b_{1}^{2} c_{1}+4 a_{2} b_{1} b_{2}^{2}\right. \\
& +6 a_{2} b_{1} b_{2} c_{1}+4 a_{2} b_{2}^{2} c_{1}-b_{1}^{2} b_{2}^{2} \hat{w}_{1}-3 b_{1}^{2} b_{2}^{2} \hat{w}_{2}-2 b_{1}^{2} b_{2}^{2} \hat{w}_{2}-2 b_{1}^{2} b_{2} c_{1} \hat{w}_{1} \\
& \left.-2 b_{1}^{2} b_{2} c_{1} \hat{w}_{2}-4 b_{1} b_{2}^{3} \hat{w}_{2}-2 b_{1} b_{2}^{2} c_{1} \hat{w}_{1}-6 b_{1} b_{2}^{2} c_{1} \hat{w}_{2}-4 b_{2}^{3} c_{1} \hat{w}_{2}\right\}\left\{6 b_{1}^{2} b_{2}^{2}\right. \\
& +4 b_{1}^{2} b_{2} c_{1}+4 b_{1}^{2} b_{2} c_{2}+4 b_{1}^{2} c_{1} c_{2}+8 b_{1} b_{2}^{3}+12 b_{1} b_{2}^{2} c_{1}+4 b_{1} b_{2}^{2} c_{2}+8 b_{1} b_{2} c_{1} c_{2} \\
& \left.+8 b_{2}^{3} c_{1}+4 b_{2}^{2} c_{1} c_{2}\right\}^{-1} .
\end{aligned}
$$

\subsection{Numerical illustrations}

We aim, in this section, at quantifying numerically the impact of local market RESbased generations, transmission and local market capacity constraints, on the PoA and on the existence of a market equilibrium in Subsection 4.3.1. The impact of simultaneous versus sequential market clearings on the PoA and optimal bidding strategy is analyzed in Subsection 4.3.2. Imperfect information is included in Subsection 4.3.3. 
Finally, we discuss the impact of inter-temporal constraints on the existence of an equilibrium and prove numerically that there exists an optimal upper-limit on the generators' bid length in Subsection 4.3.4.

\subsubsection{Impact of RES-based generation, transmission and local mar- ket capacity constraints}

In this subsection, we quantify numerically the impact of RES-based generation on the efficiency of decentralized market design, using the PoA as performance measure. Then, we focus on the analysis of the impact of transmission line and local market capacity limits, on the existence of an equilibrium for the decentralized market design.

Impact of RES-based generation on the efficiency of the decentralized market design: We want to quantify the impact of RES-based generation, $\hat{w}_{1}$ and $\hat{w}_{2}$, on the PoA, assuming that the local markets are cleared simultaneously. The parameters are set as follows $a_{1}=a_{2}=8, b_{1}=0.3, b_{2}=0.7, c_{1}=10, c_{2}=20$. In Figure 1, we have represented the PoA as a function of local RES-based generations. We observe that the PoA always remains in the range of values $[1 ; 1.025]$ and is exactly 1 in case where RES-based generation is the highest. This means that efficiency loss caused by the introduction of anticipatory behavior is the smallest for high shares of renewables. In other words, the decentralized market design output coincides with the centralized market design output when the total RES-based generation is high. A possible interpretation is that the increase of RES-based generation implies a decrease of the market price and, a fortiori, a decrease in the conventional generators' bids (that are linear in $D^{-1}(Q)$ under simultaneous market clearing). Such a situation implies less ability for conventional generators to affect pricing outcomes. These results may be in favor of fully-decentralized market designs, i.e., peer-to-peer energy trading, were no national MO is involved and local MOs/generators trade bilaterally energy with one another [21], for high penetration of renewables.

Impact of transmission line finite capacity and local market capacity constraints: We now assume that there is a single transmission line that interconnects the local market nodes, with a finite capacity. We want to check to what extent line capacity constraint might prevent the emergence of an equilibrium [3, 35]. Such effects have already been studied using a game theoretical approach in [3]. Borenstein et al. proved that there is an impact of capacity of transmission lines on the degree to which generators in different locations compete with one another. They demonstrated that there is a threshold level of capacity, above which two otherwise isolated markets are effectively merged. In case where the line capacity is below this threshold, they proved analytically that line capacity has a clear effect on the output of both generators: increases in the line capacity cause a monotonic decline in the expected market price and monotonic increase in expected quantities produced up to the point that the unconstrained market equilibrium is reached. Assuming that transmission capacity holds, the set $\mathcal{X}$ includes a constraint of the form $-f \leq h_{1} r_{1}+h_{2} r_{2} \leq f$. The shift-factor vector $\boldsymbol{H}$ depends on the admittances of the transmission line. We set the reactance to the line to $2.10^{-3}$ and the bus admittance matrix is $\left(\begin{array}{cc}1000 & -1000 \\ -1000 & 1000\end{array}\right)$.

Following [37], this implies that the associated shift-factor vector is $\boldsymbol{H}=\left(\begin{array}{ll}1 & 0\end{array}\right)$ assuming power flow is in the direction from bus 1 to 2 and bus 2 is the reference. 


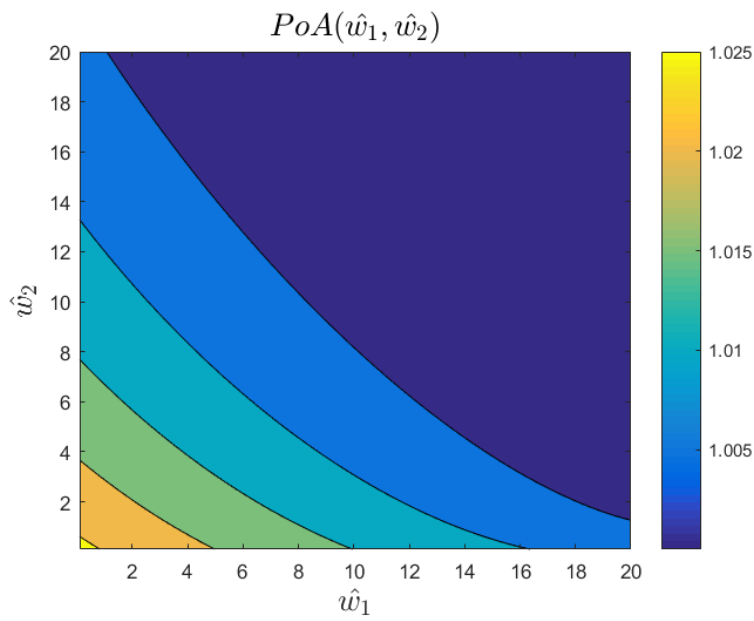

Figure 1: We evaluate numerically the impact of RES-based generation on the Price of Anarchy (PoA) for decentralized market design and simultaneous clearings of the local markets.

In Figure 2, we have represented $\boldsymbol{H} \boldsymbol{r}^{c}$ in (a) and $\boldsymbol{H} \boldsymbol{r}^{\star}$ in (b), i.e., the local market exchanges evaluated in the optimum (centralized design) and in the equilibrium (decentralized design), as functions of RES-based generation on the local markets, $\hat{w}_{1}$ and $\hat{w}_{2}$. If we choose the line transmission capacity so that $f=5$, the set of $\left(\hat{w}_{1}, \hat{w}_{2}\right) \in \mathbb{R}_{+}^{2}$ for which there exists an equilibrium is the subspace of $\mathbb{R}_{+}^{2}$ located between the two dot magenta lines. We observe that the existence of solutions for the centralized optimization problem and bilevel mathematical programming problem depends on parametrizations (here, the RES-based generations).

In addition, we assume that capacity constraints hold in each local market node, i.e., $q_{k} \leq \overline{q_{k}}, \forall k=1,2$ where $\overline{q_{k}}>0$ is the maximum production capacity available at node $k$. In Figure 3 (a), we have plotted $q_{1}^{\star}\left(\hat{w}_{1}, \hat{w}_{2}\right), q_{2}^{\star}\left(\hat{w}_{1}, \hat{w}_{2}\right)$ as functions of $\hat{w}_{1}$ and $\hat{w}_{2}$. In Figure 3 (b) we have represented $\boldsymbol{q}^{\star}$ as a function of $\hat{w}_{1}$ and $\hat{w}_{2}$ taking into account the finite capacity link constraint $\left(0 \leq r^{\star} \leq 5\right)$ already represented in Figure 2 and local market capacity constraints with $\overline{q_{1}}=\overline{q_{2}}=0.19$. The magenta area delineates the set of $\hat{w}_{1}$ and $\hat{w}_{2}$ for which equilibrium exists.

Based on these numerical illustrations, we observe that equilibrium satisfying finite transmission line capacity and local market capacity constraints might exist only for high RES shares. Otherwise, unstable behaviors will be observed which would be in favor of a more centralized design.

\subsubsection{Simultaneous versus sequential market clearing}

In this subsection, we assume the the local market 1 has lower priority than the local market 2, which implies that $M O_{2}$ announces his clearing price $p_{2}($.$) before M O_{1}$, who will announce $p_{1}($.$) later. We observe in Figure 4$ that the optimal simple bid of the generator in local market 1 is inferior under sequential market clearing than under simultaneous market clearing, for any value of RES-based generation, $\hat{w}_{1}, \hat{w}_{2}$. This 


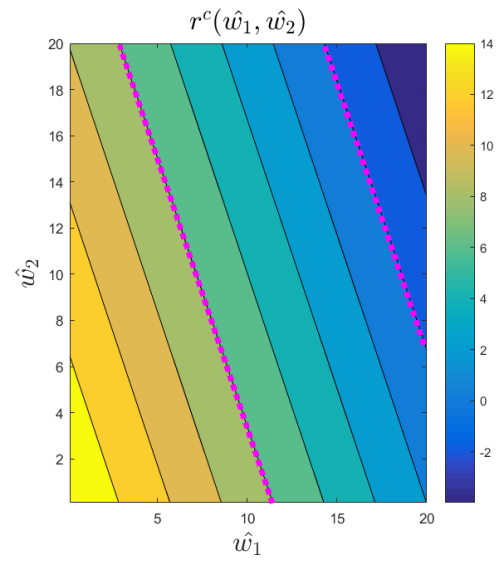

(a)

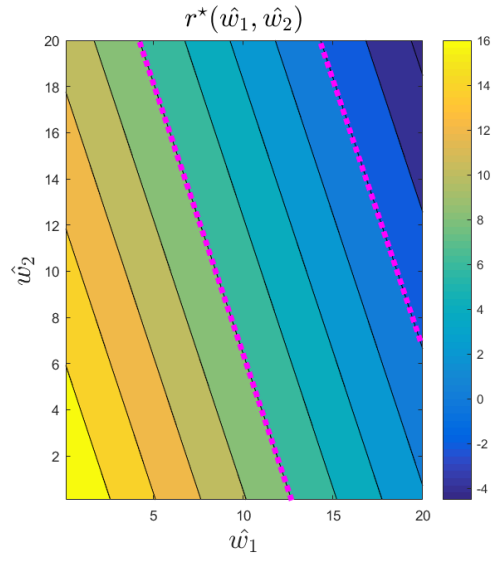

(b)

Figure 2: We represent parametric feasible sets for the local market exchanges $\boldsymbol{r}(\boldsymbol{t})$ as functions of RES-based generations for centralized (a) and decentralized market designs (b).

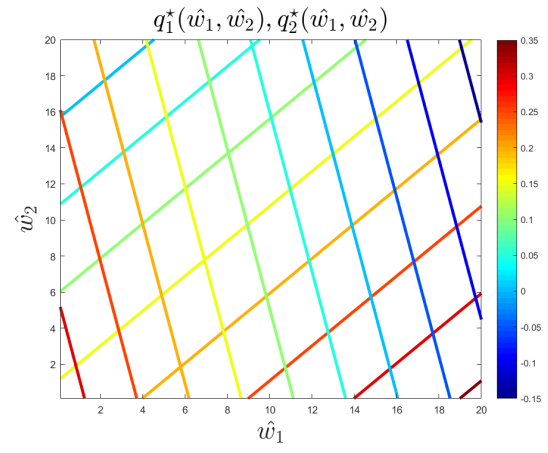

(a)

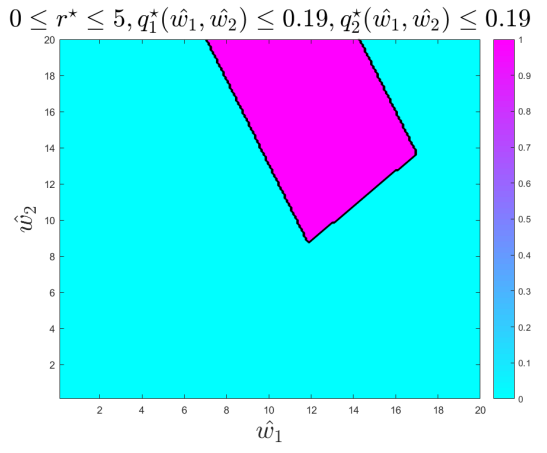

(b)

Figure 3: In (a) we represent each local market generator optimal block bid as a function of local RES-based generation, $\hat{w}_{1}, \hat{w}_{2}$. Incorporating transmission capacity $0 \leq r^{\star} \leq 5$ and local market generation capacity constraints with $\overline{q_{1}}=\overline{q_{2}}=0.19$, we delineate the set of RES-based generation where equilibrium exists in (b).

observation coincides with the result obtained in Proposition 5. In local market 2, the reverse relation holds, i.e., the optimal bid under simultaneous market clearing is lower than the optimal bid under sequential market clearing for $\hat{w}_{1} \leq 9.5, \forall \hat{w}_{2} \leq 10$ and for $\hat{w}_{1} \geq 9.5, \hat{w}_{2} \leq 5$. But for $\hat{w}_{1} \geq 9.5$ and $\hat{w}_{2} \geq 5$, the optimal bid for the generator in local market 2 is larger under simultaneous market clearing than under sequential market clearing. This numerical result illustrates that incentives for the generator in the highest priority market to bid higher quantities under simultaneous market clearing than under sequential is highly dependent on the RES-based generation. 

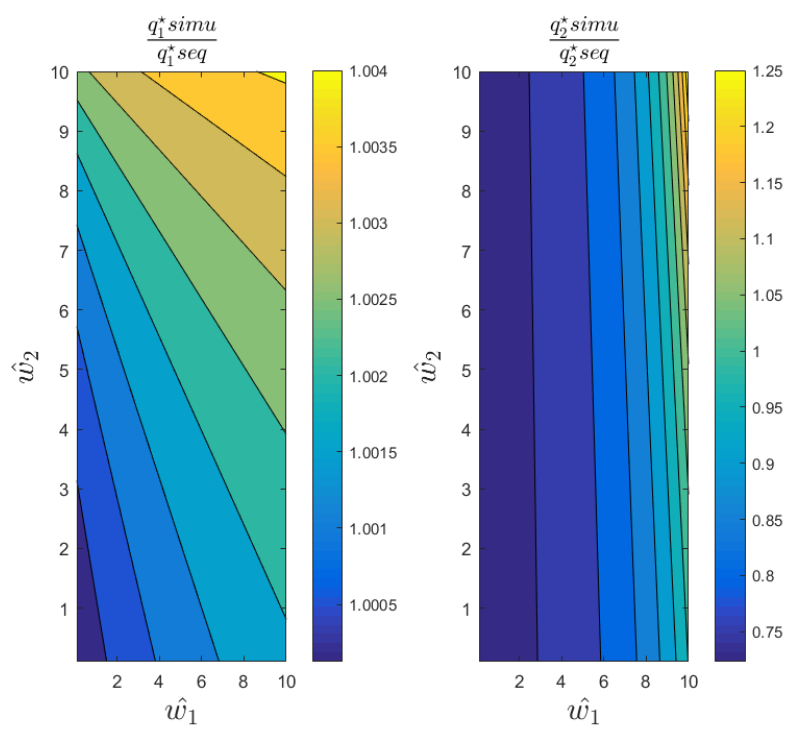

Figure 4: We compare the ratio of optimal bids under sequential and simultaneous local market clearings in local market 1 (left) and local market 2 (right).

\subsubsection{Impact of imperfect information}

To compute the closed form expressions of the optimal bids of each generator as introduced in Subsection 4.2, generators should share their (estimated) RES-based generation with all the market operators (both local and national) and the other generators, something for which they might have no a priori incentives. To check whether (RES-based generation) information sharing might be profitable for the generators, we assume that each local $\mathrm{MO}_{k}, k=1,2$, makes the estimate that $\xi_{k} \hat{w}_{l}, l=1,2, l \neq k$, renewable power is produced on the other local market, with $\xi_{k} \geq 0$ a distortion factor. Note that $\xi_{k}=1$ means that full information is disclosed, which implies that $\mathrm{MO}_{k}$ correctly guesses the RES-based generation on local market $l=1,2, l \neq k$.

We have represented in Figure 5, the PoA as a function of the distortion factors for both simultaneous and sequential market clearings, assuming that the estimated RES-based generation on each local market are set so that $\hat{w}_{1}=8$ and $\hat{w}_{2}=10$. Furthermore, we assume that under centralized market design, perfect information holds, i.e., local market RES-based generations are common knowledge. We observe in Figure 5 that for distortion factors smaller than 3, imperfect information has a limited impact on the PoA which stays exactly at 1 (meaning that the decentralized market design with imperfect information is as efficient as the centralized market design with perfect information). Identically, in [13], even with simple mechanisms to guess the bids of other market participants, results close to a perfect information benchmark can be achieved. 

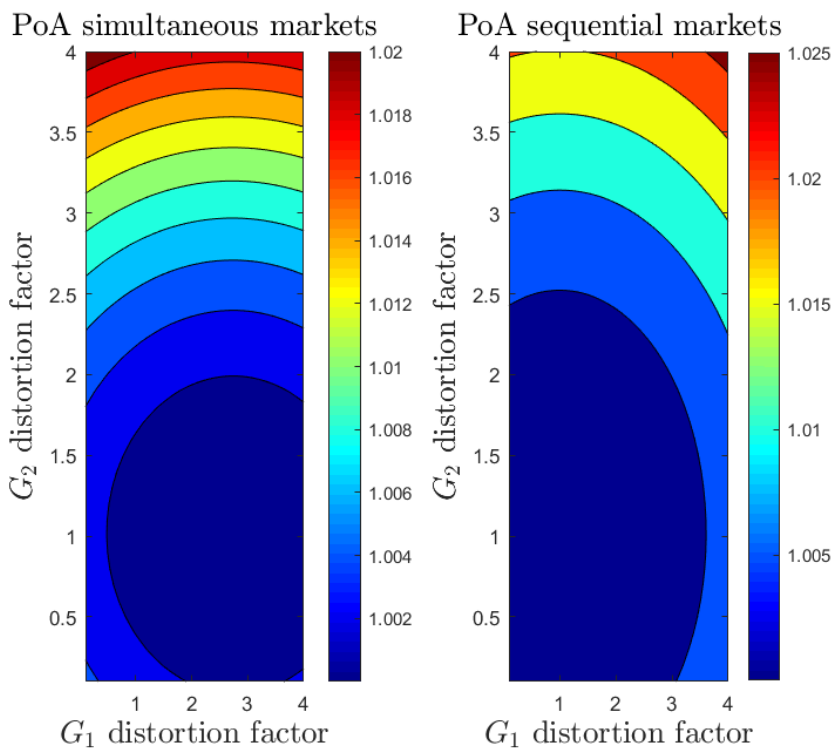

Figure 5: We quantify the impact of information distortion on the PoA for simultaneous (left) and sequential (right) market clearings.

\subsubsection{On the complexity of block bids}

The liberalized electricity market design has been extended to facilitate the trade of energy flexibility by introducing new categories of complex bids such as linked block orders, exclusive block orders and flexible orders [28, 39]. The introduction of such new categories of bids is justified by the need to take into account techno-economical aspects of generation units, currently not captured by simple quantity bids. They also generate lots of debates regarding possible trade-offs between an increase of complexity in the bid definition and the guarantee of the efficiency of market operation.

Inter-temporal constraints: In this paper, we consider quantity offers that are bidded on the energy market and take the form of (linked) block bids, i.e., length $t_{H}$ vectors subject to inter-temporal constraints that are accepted in full. Inter-temporal constraints might capture dynamic constraints such as ramping or start-up costs constraints. In the framework of this numerical illustration and for the sake of simplicity, the RES-based generation on market 2 on the time period $[t ; t+1]$ is fixed, i.e., $\hat{w}_{2}(t+1)=\hat{w}_{2}(t)=8$ and we focus on high wind share penetration. In Figure 6 left, we have represented $\frac{q_{1}^{\star}(t+1)}{q_{1}^{\star}(t)}$ as a function of the local market RES-based generation at time periods $t$ and $t+1$, i.e., $\hat{w}_{1}(t), \hat{w}_{1}(t+1)$. We observe that in case of a significant decrease in the RES-based generation, i.e., if $\hat{w}_{1}(t)>>\hat{w}_{1}(t+1)$ then, $q_{1}^{\star}(t+1)>>q_{1}^{\star}(t)$ meaning that the optimal quantity bidded by the generator in the local market is larger at $t+1$ than at $t$. Otherwise, i.e., in case of significant increase in the RES-based generation $\left(\hat{w}_{1}(t)<<\hat{w}_{1}(t+1)\right)$ then, $q_{1}^{\star}(t+1)<<q_{1}^{\star}(t)$ meaning 
that the optimal quantity bidded by the generator in local market 1 is smaller at $t+1$ than at $t$. If we take into account additional transmission line capacity constraint and local market capacity constraints, we observe in Figure 6 right, that equilibrium exists only for $\hat{w}_{1}(t+1) \geq 7$ i.e., only under high share of renewables in the local market.
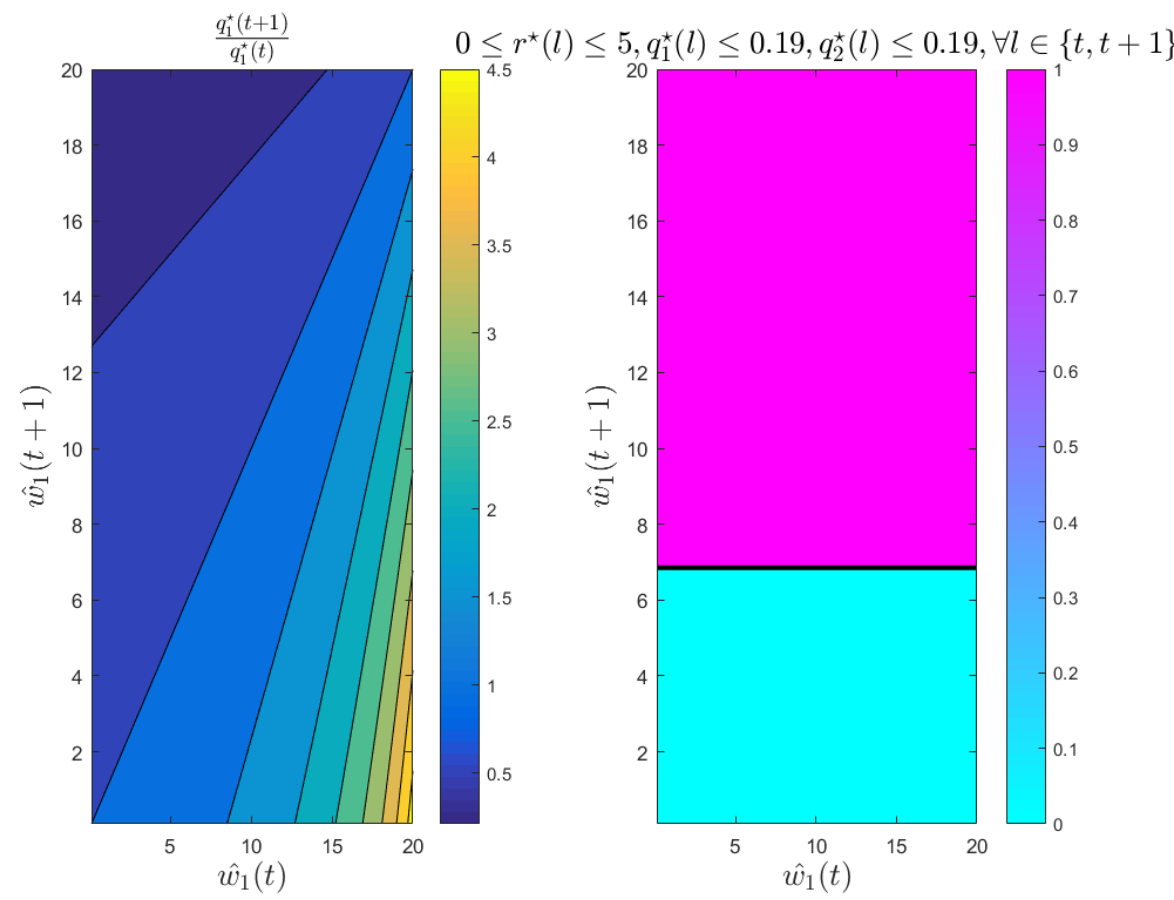

Figure 6: We represent the impact of a change (increase/decrease) in RES-based generation over two consecutive time periods on the ratio between two optimal consecutive simple bids (left) and on the existence of a market equilibrium taking into account transmission link and capacity constraints (right).

Optimal bid size: We want to optimize numerically the length of the block quantity offers submitted by the generators in the local markets. RES-based generations are based on the values provided on the ELIA website http://www.elia.be/en/ grid-data/data-download. We make the assumption that local market 1 RES-based generation is provided by an offshore wind producer only, whereas on local market 2 the RES-based generation relies mainly on onshore wind farms.

In Figure 7, we have represented the social welfare as a function of the length of the bid, evaluated in number of quarter hours (15 minutes), under centralized and decentralized market designs. The decentralized market design may involve either simultaneous or sequential market clearings of the local markets. We observe that a first threshold is reached around 190 quarters (i.e., after two consecutive days) and a third threshold is reached after 270 quarters (i.e, before three consecutive days). 
These results are therefore in favor of the existence of a maximum bid length, i.e., an upper-limit on $t_{H}$. We observe in addition in Figure 7 that the social welfare (in Equation (11)) is higher under simultaneous local market clearing than under sequential market clearing.

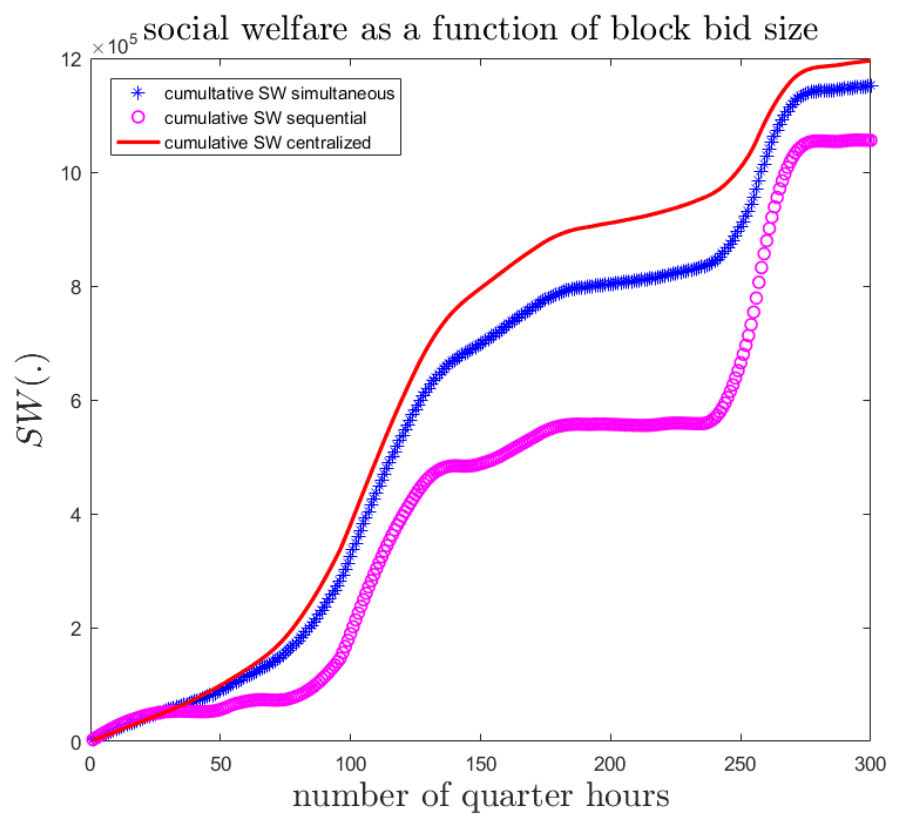

Figure 7: Impact of the block bid size on the social welfare under centralized and decentralized market designs (with either simultaneous market clearing or sequential market clearing of the local markets).

\section{Conclusion}

In this paper, we have modeled interactions between local and national Market Operators for two market designs: a centralized one, formulated as a standard constrained optimization problem, and a decentralized one, formulated as a Stackelberg game where strategic generators, who have market power, act as leaders anticipating the exchanges between the local markets, i.e., the locational marginal prices and transmission flows in line. Both cases where local markets are cleared simultaneously or sequentially are considered. We have quantified the loss of efficiency caused by the generators' anticipation through the Price of Anarchy (PoA). We have proved that the Stackelberg game, formulated as a bilevel mathematical programming problem, can be reformulated as a non linear Complementarity Problem and admits a unique Nash equilibrium in case of simple bids. The result is generalized to block bids, i.e., length $t_{H}$ quantity vectors that are linked by inter-temporal constraints, using Complementarity Theory. A case study highlights the impact of transmission line and 
local market generation capacities, as well as RES-based generation impacts, on the existence of a market equilibrium. The impact of distortion caused by imperfect information on local RES-based generation on the PoA is also quantitatively assessed. Numerical illustrations highlight that (i) decentralized market design is as efficient as the centralized market design under high share of renewables, that (ii) transmission and local market generation capacities have an impact on the existence of a market equilibrium, that (iii) imperfect information has only a limited impact on the decentralized market design efficiency compared with the centralized one, and that (iv) for each market design, there exists an optimal size for the block bids that maximizes the social welfare. Finally simultaneous market clearing gives rises to larger social welfare than sequential market clearing.

\section{References}

[1] Barquin J., Vazquez M., Cournot equilibrium computation on electricity networks, 2-nd International workshop on liberalization and modernization of power systems, congestion management problems, 2003

[2] Bose S., Cai D., Low S., Wierman A., The Role of a Market Maker in Networked Cournot Competition, Proc. 53rd IEEE conference on Decision and Control, pages 4479-4484, 2014

[3] Borenstein S., Bushnell J., Stoft S., The competitive effects of transmission capacity in a deregulated electricity industry, RAND Journal of Economics, vol. 31, no. 2, pp. 294-325, 2000

[4] Cardell J., Hitt C. C., Hogan W. W., Market power and strategic interactions in electricity networks, Resource and Energy Economics, vol. 19, Issues 1-2, pp. 25-60, 1996

[5] Chao H.-P., Peck S. C., A market mechanism for electric power transmission, Journal of Regulatory Economics, vol. 10, Issue 1, pp. 25-60, 1996

[6] Colson B., Marcotte P., Savard G., An overview of bilevel optimization, Annals of Operations Research, volume 153, pages 235-256, 2007

[7] Dempe S., Annotated Bibliography on Bilevel Programming and Mathematical Programs with Equilibrium Constraints, Optimization: A Journal of Mathematical Programming and Operations Research, volume 52, issue 3, pages 333-359, 2010

[8] Dempe S., Kalashnikov V., Perez-Valdes G. A., Kalashnikova N., Bilevel Programming Problems: Theory, Algorithms and Applications to Energy Networks, Springer, Energy Systems, 2015

[9] Dempe S., Dutta J., Is Bilevel Programming a Special Case of a Mathematical Program with Complementarity Constraints?, Mathematical Programming, volume 131, Issue 1, pages 37-48, 2012

[10] Economides N., Tag J., Net Neutrality on the Internet: A Two-sided Market Analysis, Information Economics and Policy, vol. 24, pp. 91-104, 2012

[11] Ehrenmann A., Neuhoff K., A comparison of electricity market designs in networks, Operations Research, vol. 57, Issue 2, pp. 274-286, 2009

[12] Gabriel S. A., Conejo A. J., Fuller J. D., Hobbs B. F., Ruiz C., Complementarity Modeling in Energy Markets, International Series in Operations Research \& Management Science, 2013 
[13] Gonzalez Vaya M., Andersson G., Optimal Bidding Strategy of a Plug-in Electric Vehicle Aggregator in Day-ahead Electricity Markets, Proc. European Energy Market (EEM) conference, 2013

[14] Karamardian S., The Complementarity Problem, Mathematical Programming, volume 2, pages 107-129, 1972

[15] Kolstad C. D., Mathiesen L., Computing Cournot-Nash Equilibria, Operations Research, volume 39, pages 739-748, 1991

[16] Koutsoupias E., Papadimitriou C. H., Worst-case equilibria, Proc. 16-th Annual Symposium on Theoretical Aspects of Computer Science (STACS), volume 1563, 1999

[17] Kulkarni A. A., Shanbhag U. V., A Shared-Constraint Approach to Multi-Leader Multi-Follower Games, Set-Valued and Variational Analysis, volume 22, Issue 4, pages 691-720, 2014

[18] Le Cadre H., On the Efficiency of Electricity Markets, Proc. EEM, 2017

[19] Le Cadre H., Papavasiliou A., Smeers Y., Wind Farm Portfolio Optimization, European Journal of Operational Research (EJOR), volume 247, issue 2, pages 560574,2015

[20] Lieberman M. B., Montgomery D. B., First-Mover Advantages, Strategic Management Journal, vol. 9, Special Issue: Strategy cocntent Research, pp. 41-58, 1988

[21] Matamoros J., Gregoratti D., Dohler M., Microgrids Energy Trading in Islanding Mode, Proc. IEEE SmartGridComm 2012 Symposium, Demand Side Management, Demand Response, Dynamic Pricing, 2012

[22] Moré J. J., Rheinbold W. C., On P- and S- functions and Related Classes of $n$-dimensional Nonlinear Mappings, Linear Algebra and its applications, volume 6, pages 45-68, 1973

[23] Neuhoff K., Barquin J., Maroeska G. B., Ehrenmann A., Hobbs B., Rijkers F. A. M., Vázquez M., Network-constrained Cournot models of liberalized electricity markets: the devil is in the details, Energy Economics, vol. 27, pp. 495-525, 2005

[24] Nisan N., Roughgarden T., Tardos E., Vazirani V. V., Algorithmic Game Theory, Cambridge University Press, 2007

[25] Osborne M. J., Rubinstein A., A Course in Game Theory, MIT Press, 1994

[26] Papavasiliou A., Analysis of Distribution Locational Marginal Prices, IEEE Transactions on Smart Grid, forthcoming, 2017

[27] Perez-Arriaga I., Editor, Regulation of the Power Sector, Springer, 2013

[28] Poli D., Marracci M., Clearing procedures for day-ahead Italian electricity market: are complex bids really required?, International Journal of Energy, Issue 3, volume 5, pages 70-77, 2011

[29] Purchala K., Meeus L., Van Dommelen D., Belmans R., Usefulness of DC power flow for active power flow analysis, Proc. IEEE PES General Meeting, pages 24572462, 2005

[30] Ruiz C., Conejo A. J., Pool Strategy of a Producer With Endogenous Formation of Locational Marginal Prices, IEEE Transactions on Power Systems, volume 24, 2009 
[31] Schwalbe U., Walker P., Zermelo and the Early History of Game Theory, Games and Economic Behavior, vol. 34, no. 1, pp. 123-137, 2001

[32] Smeers Y., Oggioni G., Allevi E., Schaible S., Generalized Nash Equilibrium and Market Coupling in the European Power System, Networks and Spatial Economics, vol. 12, Issue 4, pp. 503-560, 2012

[33] Stoft S., Power System Economics: Designing Market for Power. Piscataway, NJ: IEEE Press, 2002

[34] Szidarovsky F., Yakowitz S., A new proof of the existence and uniqueness of the Cournot equilibrium, International Economic Review, volume 18, pages 787-789, 1977

[35] Xu Y., Cai D., Bose S., Wierman A., On the Efficiency of Networked Stackelberg Competition, Proc. IEEE conference on Decision and Control, 2015

[36] Yao J., Adler I., Oren S. S., Modeling and computing two-settlement oligopolistic equilibrium in a congested electricity network, Operations Research, vol. 56, no. 1, pp. $34-47,2008$

[37] California ISO, Shift Factors: Methodology and Example, CAISO Market Operations, http://www.caiso.com/docs/2004/02/13/200402131609438684.pdf, [Online December 2016]

[38] Optimate Platform, A numerical simulation platform to recommend new electricity market designs integrating massive flexible generation in Europe, http: //www.optimate-platform.eu/, [Online January 2017]

[39] Euphemia, https://www.nordpoolspot.com/globalassets/download-center/ pcr/euphemia-public-documentation.pdf, [Online September 2017] 